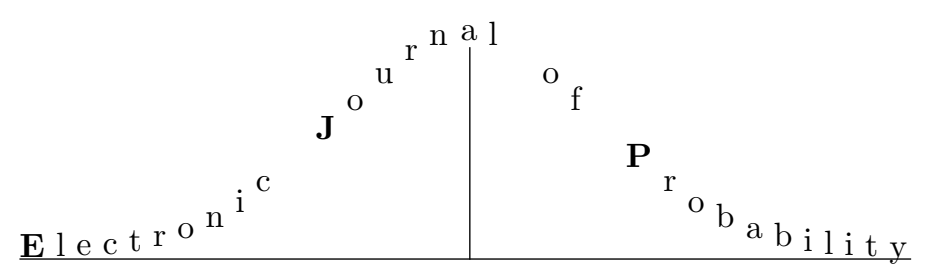

Vol. 13 (2008), Paper no. 35, pages 1035-1067.

Journal URL

http://www.math. washington.edu/ ejpecp/

\title{
Sobolev solution for semilinear PDE with obstacle under monotonicity condition
}

\author{
Anis Matoussi \\ Equipe "Statistique et Processus" \\ Université du Maine \\ Avenue Olivier Messiaen \\ 72085 LE MANS Cedex, France \\ anis.matoussi@univ-lemans.fr
}

\author{
Mingyu $\mathrm{Xu}$ \\ Institute of Applied Mathematics \\ Academy of Mathematics and Systems Science \\ CAS, Beijing, 100190 China \\ xumy@amss.ac.cn
}

\begin{abstract}
We prove the existence and uniqueness of Sobolev solution of a semilinear PDE's and PDE's with obstacle under monotonicity condition. Moreover we give the probabilistic interpretation of the solutions in term of Backward SDE and reflected Backward SDE respectively .
\end{abstract}

Key words: Backward stochastic differential equation, Reflected backward stochastic differential equation, monotonicity condition, Stochastic flow, partial differential equation with obstacle.

AMS 2000 Subject Classification: Primary 35D05, 60H10, 60H30B.

Submitted to EJP on August 29, 2005, final version accepted May 30, 2008. 


\section{Introduction}

Our approach is based on Backward Stochastic Differential Equations (in short BSDE's) which were first introduced by Bismut [5] in 1973 as equation for the adjoint process in the stochastic version of Pontryagin maximum principle. Pardoux and Peng [14] generalized the notion in 1990 and were the first to consider general BSDE's and to solve the question of existence and uniqueness in the non-linear case. Since then BSDE's have been widely used in stochastic control and especially in mathematical finance, as any pricing problem by replication can be written in terms of linear BSDEs, or non-linear BSDEs when portfolios constraints are taken into account as in El Karoui, Peng and Quenez [6].

The main motivation to introduce the non-linear BSDE's was to give a probabilistic interpretation (Feynman-Kac's formula) for the solutions of semilinear parabolic PDE's. This result was first obtained by Peng in [16], see also Pardoux and Peng [15] by considering the viscosity and classical solutions of such PDE's. Later, Barles and Lesigne [2] studied the relation between BSDE's and solutions of semi-linear PDE's in Soblev spaces. More recently Bally and Matoussi [3] studied semilinear stochastic PDEs and backward doubly SDE in Sobolev space and their probabilistic method is based on stochastic flow.

The reflected BSDE's was introduced by the five authors El Karoui, Kapoudjian, Pardoux, Peng and Quenez in [7], the setting of those equations is the following: let us consider moreover an adapted stochastic process $L:=\left(L_{t}\right)_{t \leqslant T}$ which stands for a barrier. A solution for the reflected BSDE associated with $(\xi, g, L)$ is a triple of adapted stochastic processes $\left(Y_{t}, Z_{t}, K_{t}\right)_{t \leqslant T}$ such that

$$
\left\{\begin{array}{l}
Y_{t}=\xi+\int_{t}^{T} g\left(s, \omega, Y_{s}, Z_{s}\right) d s+K_{T}-K_{t}-\int_{t}^{T} Z_{s} d B_{s}, \forall t \in[0, T], \\
Y_{t} \geqslant L_{t} \text { and } \int_{0}^{T}\left(Y_{t}-L_{t}\right) d K_{t}=0 .
\end{array}\right.
$$

The process $K$ is continuous, increasing and its role is to push upward $Y$ in order to keep it above the barrier $L$. The requirement $\int_{0}^{T}\left(Y_{t}-L_{t}\right) d K_{t}=0$ means that the action of $K$ is made with a minimal energy.

The development of reflected BSDE's (see for example [7], [10], [9]) has been especially motivated by pricing American contingent claim by replication, especially in constrained markets. Actually it has been shown by El Karoui, Pardoux and Quenez [8] that the price of an American contingent claim $\left(S_{t}\right)_{t \leqslant T}$ whose strike is $\gamma$ in a standard complete financial market is $Y_{0}$ where $\left(Y_{t}, \pi_{t}, K_{t}\right)_{t \leqslant T}$ is the solution of the following reflected BSDE

$$
\left\{\begin{array}{l}
-d Y_{t}=b\left(t, Y_{t}, \pi_{t}\right) d t+d K_{t}-\pi_{t} d W_{t}, \quad Y_{T}=\left(S_{T}-\gamma\right)^{+} \\
Y_{t} \geqslant\left(S_{t}-\gamma\right)^{+} \quad \text { and } \quad \int_{0}^{T}\left(Y_{t}-\left(S_{t}-\gamma\right)^{+}\right) d K_{t}=0
\end{array}\right.
$$

for an appropriate choice of the function $b$. The process $\pi$ allows to construct a replication strategy and $K$ is a consumption process that could have the buyer of the option. In a standard financial market the function $b(t, \omega, y, z)=r_{t} y+z \theta_{t}$ where $\theta_{t}$ is the risk premium and $r_{t}$ the spot rate to invest or borrow. Now when the market is constrained i.e. the interest rates are not the same whether we borrow or invest money then the function $b(t, \omega, y, z)=r_{t} y+z \theta_{t}-$ $\left(R_{t}-r_{t}\right)\left(y-\left(z \cdot \sigma_{t}^{-1} \cdot \mathbf{1}\right)\right)^{-}$where $R_{t}$ (resp. $\left.r_{t}\right)$ is the spot rate to borrow (resp. invest) and $\sigma$ the volatility. 
Partial Differential Equations with obstacles and their connections with optimal control problems have been studied by Bensoussan and Lions [4]. They study such equations in the point of view of variational inequalities. In a recent paper, Bally, Caballero, El Karoui and Fernandez [1] studied the the following semilinear PDE with obstacle

$$
\left(\partial_{t}+\mathcal{L}\right) u+f\left(t, x, u, \sigma^{*} \nabla u\right)+\nu=0, u \geqslant h, u_{T}=g,
$$

where $h$ is the obstacle. The solution of such equation is a pair $(u, \nu)$ where $u$ is a function in $\mathbf{L}^{2}([0, T], \mathcal{H})$ and $\nu$ is a positive measure concentrated on the set $\{u=h\}$. The authors proved the uniqueness and existence for the solution to this PDE when the coefficient $f$ is Lipschitz and linear increasing on $(y, z)$, and gave the probabilistic interpretation (Feynman-Kac formula) for $u$ and $\nabla u$ by the solution $(Y, Z)$ of the reflected BSDE (in short RBSDE). They prove also the natural relation between Reflected BSDE's and variational inequalities and prove uniqueness of the solution for such variational problem by using the relation between the increasing process $K$ and the measure $\nu$. This is also a point of view in this paper.

On the other hand, Pardoux [13] studied the solution of a BSDE with a coefficient $f(t, \omega, y, z)$, which satisfies only monotonicity, continuous and general increasing conditions on $y$, and a Lipschitz condition on $z$, i.e. for some continuous, increasing function $\varphi: \mathbb{R}_{+} \rightarrow \mathbb{R}_{+}$, and real numbers $\mu \in \mathbb{R}, k>0, \forall t \in[0, T], \forall y, y^{\prime} \in \mathbb{R}^{n}, \forall z, z^{\prime} \in \mathbb{R}^{n \times d}$,

$$
\begin{aligned}
|f(t, y, 0)| & \leqslant|f(t, 0,0)|+\varphi(|y|), \text { a.s.; } \\
\left\langle y-y^{\prime}, f(t, y, z)-f\left(t, y^{\prime}, z\right)\right\rangle & \leqslant \mu\left|y-y^{\prime}\right|^{2}, \text { a.s.; } \\
\left|f(t, y, z)-f\left(t, y, z^{\prime}\right)\right| & \leqslant k\left|z-z^{\prime}\right|, \text { a.s.. }
\end{aligned}
$$

In the same paper, he also considered the PDE whose coefficient $f$ satisfies the monotonicity condition (1), proved the existence of a viscosity solution $u$ to this PDE and gave its probabilistic interpretation via the solution of the corresponding BSDE. More recently, Lepeltier, Matoussi

and $\mathrm{Xu}[12]$ proved the existence and uniqueness of the solution for the reflected BSDE under the monotonicity condition.

In our paper, we study the Sobolev solutions of the PDE and also the PDE with continuous obstacle under the monotonicity condition (1). Using penalization method, we prove the existence of the solution and give the probabilistic interpretation of the solution $u$ and $\nabla u(\operatorname{resp} .(u, \nabla u, \nu))$ by the solution $(Y, Z)$ of backward SDE (resp. the solution $(Y, Z, K)$ of reflected backward SDE). Furthermore we use equivalence norm results and a stochastic test function to pass from the solution of PDE's to the one of BSDE's in order to get the uniqueness of the solution.

Our paper is organized as following: in section 2, we present the basic assumptions and the definitions of the solutions for PDE and PDE with obstacle, then in section 3, we recall some useful results from [3]. We will prove the main results for PDE and PDE with continuous barrier under monotonicity condition in section 4 and 5 respectively. Finally, we prove an analogue result to Proposition 2.3 in [3] under the monotonicity condition, and we also give a priori estimates for the solution of the reflected BSDE's.

\section{Notations and preliminaries}

Let $(\Omega, \mathcal{F}, P)$ be a complete probability space, and $B=\left(B_{1}, B_{2}, \cdots, B_{d}\right)^{*}$ be a $d$-dimensional Brownian motion defined on a finite interval $[0, T], 0<T<+\infty$. Denote by $\left\{\mathcal{F}_{s}^{t} ; t \leqslant s \leqslant T\right\}$ 
the natural filtration generated by the Brownian motion $B$ :

$$
\mathcal{F}_{s}^{t}=\sigma\left\{B_{s}-B_{t} ; t \leqslant r \leqslant s\right\} \cup \mathcal{F}_{0},
$$

where $\mathcal{F}_{0}$ contains all $P$-null sets of $\mathcal{F}$.

We will need the following spaces for studying BSDE or reflected BSDE. For any given $n \in \mathbb{N}$ :

- $\mathbf{L}_{n}^{2}\left(\mathcal{F}_{s}^{t}\right)$ : the set of $n$-dimensional $\mathcal{F}_{s}^{t}$-measurable random variable $\xi$, such that $E\left(|\xi|^{2}\right)<$ $+\infty$.

- $\mathbf{H}_{n \times m}^{2}(t, T)$ : the set of $\mathbb{R}^{m \times n}$-valued $\mathcal{F}_{s}^{t}$-predictable process $\psi$ on the interval $[t, T]$, such that $E \int_{t}^{T}\|\psi(s)\|^{2} d s<+\infty$.

- $\mathbf{S}_{n}^{2}(t, T)$ : the set of $n$-dimensional $\mathcal{F}_{s}^{t}$-progressively measurable process $\psi$ on the interval $[t, T]$, such that $E\left(\sup _{t \leqslant s \leqslant T}\|\psi(s)\|^{2}\right)<+\infty$.

- $\mathbf{A}^{2}(t, T):=\left\{K: \Omega \times[t, T] \rightarrow \mathbb{R}, \mathcal{F}_{s}^{t}\right.$-progressively measurable increasing RCLL processes with $\left.K_{t}=0, E\left[\left(K_{T}\right)^{2}\right]<\infty\right\}$.

Finally, we shall denote by $\mathcal{P}$ the $\sigma$-algebra of predictable sets on $[0, T] \times \Omega$. In the realvalued case, i.e., $n=1$, these spaces will be simply denoted by $\mathbf{L}^{2}\left(\mathcal{F}_{s}^{t}\right), \mathbf{H}^{2}(t, T)$ and $\mathbf{S}^{2}(t, T)$, respectively.

For the sake of the Sobolev solution of the PDE, the following notations are needed:

- $C_{b}^{m}\left(\mathbb{R}^{d}, \mathbb{R}^{n}\right)$ : the set of $C^{m}$-functions $f: \mathbb{R}^{d} \rightarrow \mathbb{R}^{n}$, whose partial derivatives of order less that or equal to $m$, are bounded. (The functions themselves need not to be bounded)

- $C_{c}^{1, m}\left([0, T] \times \mathbb{R}^{d}, \mathbb{R}^{n}\right)$ : the set of continuous functions $f:[0, T] \times \mathbb{R}^{d} \rightarrow \mathbb{R}^{n}$ with compact support, whose first partial derivative with respect to $t$ and partial derivatives of order less or equal to $m$ with respect to $x$ exist.

- $\rho: \mathbb{R}^{d} \rightarrow \mathbb{R}$, the weight, is a continuous positive function which satisfies $\int_{\mathbb{R}^{d}} \rho(x) d x<\infty$.

- $\mathbf{L}^{2}\left(\mathbb{R}^{d}, \rho(x) d x\right)$ : the weighted $\mathbf{L}^{2}$-space with weight function $\rho(x)$, endowed with the norm

$$
\|u\|_{\mathbf{L}^{2}\left(\mathbb{R}^{d}, \rho\right)}^{2}=\int_{\mathbb{R}^{d}}|u(x)|^{2} \rho(x) d x
$$

We assume:

Assumption 2.1. $g(\cdot) \in \mathbf{L}^{2}\left(\mathbb{R}^{d}, \rho(x) d x\right)$.

Assumption 2.2. $f:[0, T] \times \mathbb{R}^{d} \times \mathbb{R}^{n} \times \mathbb{R}^{n \times d} \rightarrow \mathbb{R}^{n}$ is measurable in $(t, x, y, z)$ and

$$
\int_{0}^{T} \int_{\mathbb{R}^{d}}|f(t, x, 0,0)|^{2} \rho(x) d x d t<\infty .
$$

Assumption 2.3. $f$ satisfies increasing and monotonicity condition on $y$, for some continuous increasing function $\varphi: \mathbb{R}_{+} \rightarrow \mathbb{R}_{+}$, real numbers $k>0, \mu \in \mathbb{R}$ such that $\forall\left(t, x, y, y^{\prime}, z, z^{\prime}\right) \in$ $[0, T] \times \mathbb{R}^{d} \times \mathbb{R}^{n} \times \mathbb{R}^{n} \times \mathbb{R}^{n \times d} \times \mathbb{R}^{n \times d}$ 
(i) $|f(t, x, y, z)| \leqslant|f(t, x, 0, z)|+\varphi(|y|)$,

(ii) $\left|f(t, x, y, z)-f\left(t, x, y, z^{\prime}\right)\right| \leqslant k\left|z-z^{\prime}\right|$,

(iii) $\left\langle y-y^{\prime}, f(t, x, y, z)-f\left(t, x, y^{\prime}, z\right)\right\rangle \leqslant \mu\left|y-y^{\prime}\right|^{2}$,

(iv) $y \rightarrow f(t, x, y, z)$ is continuous.

For the PDE with obstacle, we consider that $f$ satisfies assumptions 2.2 and 2.3 , for $n=1$.

Assumption 2.4. The obstacle function $h \in C\left([0, T] \times \mathbb{R}^{d} ; \mathbb{R}\right)$ satisfies the following conditions: there exists $\kappa \in \mathbb{R}, \beta>0$, such that $\forall(t, x) \in[0, T] \times \mathbb{R}^{d}$

(i) $\varphi\left(e^{\mu t} h^{+}(t, x)\right) \in \mathbf{L}^{2}\left(\mathbb{R}^{d} ; \rho(x) d x\right)$,

(ii) $|h(t, x)| \leqslant \kappa\left(1+|x|^{\beta}\right)$,

here $h^{+}$is the positive part of $h$.

Assumption 2.5. $b:[0, T] \times \mathbb{R}^{d} \rightarrow \mathbb{R}^{d}$ and $\sigma:[0, T] \times \mathbb{R}^{d} \rightarrow \mathbb{R}^{d \times d}$ satisfy

$$
b \in C_{b}^{2}\left(\mathbb{R}^{d} ; \mathbb{R}^{d}\right) \quad \text { and } \quad \sigma \in C_{b}^{3}\left(\mathbb{R}^{d} ; \mathbb{R}^{d \times d}\right) .
$$

We first study the following PDE

$$
\left\{\begin{array}{l}
\left(\partial_{t}+\mathcal{L}\right) u+F(t, x, u, \nabla u)=0, \quad \forall(t, x) \in[0, T] \times \mathbb{R}^{d} \\
u(x, T)=g(x), \quad \forall x \in \mathbb{R}^{d}
\end{array}\right.
$$

where $F:[0, T] \times \mathbb{R}^{d} \times \mathbb{R}^{n} \times \mathbb{R}^{n \times d} \rightarrow \mathbb{R}$, such that

$$
F(t, x, u, p)=f\left(t, x, u, \sigma^{*} p\right)
$$

and

$$
\mathcal{L}=\sum_{i=1}^{d} b_{i} \frac{\partial}{\partial x_{i}}+\frac{1}{2} \sum_{i, j=1}^{d} a_{i, j} \frac{\partial^{2}}{\partial x_{i} \partial x_{j}},
$$

$a:=\sigma \sigma^{*}$. Here $\sigma^{*}$ is the transposed matrix of $\sigma$.

In order to study the weak solution of the PDE, we introduce the following space

$$
\mathcal{H}:=\left\{u \in \mathbf{L}^{2}\left([0, T] \times \mathbb{R}^{d}, d s \otimes \rho(x) d x\right) \mid \sigma^{*} \nabla u \in \mathbf{L}^{2}\left(\left([0, T] \times \mathbb{R}^{d}, d s \otimes \rho(x) d x\right)\right\}\right.
$$

endowed with the norm

$$
\|u\|^{2}:=\int_{\mathbb{R}^{d}} \int_{0}^{T}\left[|u(s, x)|^{2}+\left|\left(\sigma^{*} \nabla u\right)(s, x)\right|^{2}\right] \rho(x) d s d x .
$$

Definition 2.1. We say that $u \in \mathcal{H}$ is the weak solution of the PDE associated to $(g, f)$, if (i) $\|u\|^{2}<\infty$,

(ii) for every $\phi \in C_{c}^{1, \infty}\left([0, T] \times \mathbb{R}^{d}\right)$

$$
\int_{t}^{T}\left(u_{s}, \partial_{t} \phi\right) d s+(u(t, \cdot), \phi(t, \cdot))-(g(\cdot), \phi(\cdot, T))+\int_{t}^{T} \mathcal{E}\left(u_{s}, \phi_{s}\right) d s=\int_{t}^{T}\left(f\left(s, \cdot, u_{s}, \sigma^{*} \nabla u_{s}\right), \phi_{s}\right) d s .
$$


where $(\phi, \psi)=\int_{\mathbb{R}^{d}} \phi(x) \psi(x) d x$ denotes the scalar product in $\mathbf{L}^{2}\left(\mathbb{R}^{d}, d x\right)$ and

$$
\mathcal{E}(\psi, \phi)=\int_{\mathbb{R}^{d}}\left(\left(\sigma^{*} \nabla \psi\right)\left(\sigma^{*} \nabla \phi\right)+\phi \nabla\left(\left(\frac{1}{2} \sigma^{*} \nabla \sigma+b\right) \psi\right)\right) d x
$$

is the energy of the system of our PDE which corresponds to the Dirichlet form associated to the operator $\mathcal{L}$ when it is symmetric. Indeed $\mathcal{E}(\psi, \phi)=-(\phi, \mathcal{L} \psi)$.

The probabilistic interpretation of the solution of PDE associated with $g, f$, which satisfy Assumption 2.1-2.3 was firstly studied by (Pardoux [13]), where the author proved the existence of a viscosity solution to this PDE, and gave its probabilistic interpretation. In section 4 , we consider the weak solution to PDE (2) in Sobolev space, and give the proof of the existence and uniqueness of the solution as well as the probabilistic interpretation.

In the second part of this article, we will consider the obstacle problem associated to the PDE (2) with obstacle function $h$, where we restrict our study in the one dimensional case $(n=1)$. Formulaly, The solution $u$ is dominated by $h$, and verifies the equation in the following sense : $\forall(t, x) \in[0, T] \times \mathbb{R}^{d}$

$$
\begin{aligned}
& \text { (i) }\left(\partial_{t}+\mathcal{L}\right) u+F(t, x, u, \nabla u) \leqslant 0, \quad \text { on } \quad u(t, x) \geqslant h(t, x), \\
& \text { (ii) }\left(\partial_{t}+\mathcal{L}\right) u+F(t, x, u, \nabla u)=0, \quad \text { on } \quad u(t, x)>h(t, x), \\
& \text { (iii) } u(x, T)=g(x) .
\end{aligned}
$$

where $\mathcal{L}=\sum_{i=1}^{d} b_{i} \frac{\partial}{\partial x_{i}}+\frac{1}{2} \sum_{i, j=1}^{d} a_{i, j} \frac{\partial^{2}}{\partial x_{i} \partial x_{j}}, a=\sigma \sigma^{*}$. In fact, we give the following formulation of the PDE with obstacle.

Definition 2.2. We say that $(u, \nu)$ is the weak solution of the PDE with obstacle associated to $(g, f, h)$, if

(i) $\|u\|^{2}<\infty, u \geqslant h$, and $u(T, x)=g(x)$.

(ii) $\nu$ is a positive Radon measure such that $\int_{0}^{T} \int_{\mathbb{R}^{d}} \rho(x) d \nu(t, x)<\infty$,

(iii) for every $\phi \in C_{c}^{1, \infty}\left([0, T] \times \mathbb{R}^{d}\right)$

$$
\begin{aligned}
& \int_{t}^{T}\left(u_{s}, \partial_{s} \phi\right) d s+(u(t, \cdot), \phi(t, \cdot))-(g(\cdot), \phi(\cdot, T))+\int_{t}^{T} \mathcal{E}\left(u_{s}, \phi_{s}\right) d s \\
= & \int_{t}^{T}\left(f\left(s, \cdot, u_{s}, \sigma^{*} \nabla u_{s}\right), \phi_{s}\right) d s+\int_{t}^{T} \int_{\mathbb{R}^{d}} \phi(s, x) 1_{\{u=h\}} d \nu(x, s) .
\end{aligned}
$$

\section{Stochastic flow and random test functions}

Let $\left(X_{s}^{t, x}\right)_{t \leqslant s \leqslant T}$ be the solution of

$$
\left\{\begin{array}{l}
d X_{s}^{t, x}=b\left(s, X_{s}^{t, x}\right) d s+\sigma\left(s, X_{s}^{t, x}\right) d B_{s} \\
X_{t}^{t, x}=x
\end{array}\right.
$$

where $b:[0, T] \times \mathbb{R}^{d} \rightarrow \mathbb{R}^{d}$ and $\sigma:[0, T] \times \mathbb{R}^{d} \rightarrow \mathbb{R}^{d \times d}$ satisfy Assumption 2.5 . 
So $\left\{X_{s}^{t, x}, x \in \mathbb{R}^{d}, t \leqslant s \leqslant T\right\}$ is the stochastic flow associated to the diffuse $\left\{X_{s}^{t, x}\right\}$ and denote by $\left\{\widehat{X}_{s}^{t, x}, t \leqslant s \leqslant T\right\}$ the inverse flow. It is known that $x \rightarrow \widehat{X}_{s}^{t, x}$ is differentiable (Ikeda and Watanabe [? ]). We denote by $J\left(X_{s}^{t, x}\right)$ the determinant of the Jacobian matrix of $\hat{X}_{s}^{t, x}$, which is positive, and $J\left(X_{t}^{t, x}\right)=1$.

For $\phi \in C_{c}^{\infty}\left(\mathbb{R}^{d}\right)$ we define a process $\phi_{t}: \Omega \times[0, T] \times \mathbb{R}^{d} \rightarrow \mathbb{R}$ by

$$
\phi_{t}(s, x):=\phi\left(\widehat{X}_{s}^{t, x}\right) J\left(\widehat{X}_{s}^{t, x}\right) .
$$

Following Kunita (See [11]), we know that for $v \in \mathbf{L}^{2}\left(\mathbb{R}^{d}\right)$, the composition of $v$ with the stochastic flow is

$$
\left(v \circ X_{s}^{t, \cdot}, \phi\right):=\left(v, \phi_{t}(s, \cdot)\right) .
$$

Indeed, by a change of variable, we have

$$
\left(v \circ X_{s}^{t, \cdot}, \phi\right)=\int_{\mathbb{R}^{d}} v(y) \phi\left(\widehat{X}_{s}^{t, y}\right) J\left(\widehat{X}_{s}^{t, y}\right) d y=\int_{\mathbb{R}^{d}} v\left(X_{s}^{t, x}\right) \phi(x) d x .
$$

The main idea in Bally and Matoussi [3] and Bally et al. [1], is to use $\phi_{t}$ as a test function in (2) and (3). The problem is that $s \rightarrow \phi_{t}(s, x)$ is not differentiable so that $\int_{t}^{T}\left(u_{s}, \partial_{s} \phi\right) d s$ has no sense. However $\phi_{t}(s, x)$ is a semimartingale and they proved the following semimartingale decomposition of $\phi_{t}(s, x)$ :

Lemma 3.1. For every function $\phi \in \mathbf{C}_{c}^{2}\left(\mathbb{R}^{d}\right)$,

$$
\phi_{t}(s, x)=\phi(x)-\sum_{j=1}^{d} \int_{t}^{s}\left(\sum_{i=1}^{d} \frac{\partial}{\partial x_{i}}\left(\sigma_{i j}(x) \phi_{t}(r, x)\right)\right) d B_{r}^{j}+\int_{t}^{s} \mathcal{L}^{*} \phi_{t}(r, x) d r,
$$

where $\mathcal{L}^{*}$ is the adjoint operator of $\mathcal{L}$. So

$$
d \phi_{t}(r, x)=-\sum_{j=1}^{d}\left(\sum_{i=1}^{d} \frac{\partial}{\partial x_{i}}\left(\sigma_{i j}(x) \phi_{t}(r, x)\right)\right) d B_{r}^{j}+\mathcal{L}^{*} \phi_{t}(r, x) d r
$$

Then in (2) we may replace $\partial_{s} \phi d s$ by the Itô stochastic integral with respect to $d \phi_{t}(s, x)$, and have the following proposition which allows us to use $\phi_{t}$ as a test function. The proof will be given in the appendix.

Proposition 3.1. Assume that assumptions 2.1, 2.2 and 2.3 hold. Let $u \in \mathcal{H}$ be a weak solution of PDE (2), then for $s \in[t, T]$ and $\phi \in C_{c}^{2}\left(\mathbb{R}^{d}\right)$,

$$
\begin{aligned}
& \int_{\mathbb{R}^{d}} \int_{s}^{T} u(r, x) d \phi_{t}(r, x) d x-\left(g(\cdot), \phi_{t}(T, \cdot)\right)+\left(u(s, \cdot), \phi_{t}(s, \cdot)\right)-\int_{s}^{T} \mathcal{E}\left(u(r, \cdot), \phi_{t}(r, \cdot)\right) d r \\
= & \int_{\mathbb{R}^{d}} \int_{s}^{T} f\left(r, x, u(r, x), \sigma^{*} \nabla u(r, x)\right) \phi_{t}(r, x) d r d x . \text { a.s. }
\end{aligned}
$$

Remark 3.1. Here $\phi_{t}(r, x)$ is $\mathbb{R}$-valued. We consider that in (6), the equality holds for each component of $u$. 
We need the result of equivalence of norms, which play important roles in existence proof for PDE under monotonic conditions. The equivalence of functional norm and stochastic norm is first proved by Barles and Lesigne [2] for $\rho=1$. In Bally and Matoussi [3] proved the same result for weighted integrable function by using probabilistic method. Let $\rho$ be a weighted function, we take $\rho(x):=\exp (F(x))$, where $F: \mathbb{R}^{d} \rightarrow \mathbb{R}$ is a continuous function. Moreover, we assume that there exists a constant $R>0$, such that for $|x|>R, F \in C_{b}^{2}\left(\mathbb{R}^{d}, \mathbb{R}\right)$. For instant, we can take $\rho(x)=(1+|x|)^{-q}$ or $\rho(x)=\exp \alpha|x|$, with $q>d+1, \alpha \in \mathbb{R}$.

Proposition 3.2. Suppose that assumption 2.5 hold, then there exists two constants $k_{1}, k_{2}>0$, such that for every $t \leqslant s \leqslant T$ and $\phi \in \mathbf{L}^{1}\left(\mathbb{R}^{d}, \rho(x) d x\right)$, we have

$$
k_{2} \int_{\mathbb{R}^{d}}|\phi(x)| \rho(x) d x \leqslant \int_{\mathbb{R}^{d}} E\left(\left|\phi\left(X_{s}^{t, x}\right)\right|\right) \rho(x) d x \leqslant k_{1} \int_{\mathbb{R}^{d}}|\phi(x)| \rho(x) d x,
$$

Moreover, for every $\psi \in \mathbf{L}^{1}\left([0, T] \times \mathbb{R}^{d}, d t \otimes \rho(x) d x\right)$

$$
\begin{aligned}
k_{2} \int_{\mathbb{R}^{d}} \int_{t}^{T}|\psi(s, x)| \rho(x) d s d x & \leqslant \int_{\mathbb{R}^{d}} \int_{t}^{T} E\left(\left|\psi\left(s, X_{s}^{t, x}\right)\right|\right) \rho(x) d s d x \\
& \leqslant k_{1} \int_{\mathbb{R}^{d}} \int_{t}^{T}|\psi(s, x)| \rho(x) d s d x,
\end{aligned}
$$

where the constants $k_{1}, k_{2}$ depend only on $T, \rho$ and the bounds of the first (resp. first and second) derivatives of $b$ (resp. $\sigma$ ).

This proposition is easy to get from the follwing Lemma, see Lemma 5.1 in Bally and Matoussi $[3]$.

Lemma 3.2. There exist two constants $c_{1}>0$ and $c_{2}>0$ such that $\forall x \in \mathbb{R}^{d}, 0 \leqslant t \leqslant T$

$$
c_{1} \leqslant E\left(\frac{\rho\left(t, \widehat{X}_{t}^{0, x}\right) J\left(\widehat{X}_{t}^{0, x}\right)}{\rho(x)}\right) \leqslant c_{2} .
$$

\section{Sobolev's Solutions for PDE's under monotonicity condition}

In this section we shall study the solution of the PDE whose coefficient $f$ satisfies the monotonicity condition. For this sake, we introduce the BSDE associated with $(g, f)$ : for $t \leqslant s \leqslant T$,

$$
Y_{s}^{t, x}=g\left(X_{T}^{t, x}\right)+\int_{s}^{T} f\left(r, X_{r}^{t, x}, Y_{r}^{t, x}, Z_{r}^{t, x}\right) d r-\int_{s}^{T} Z_{s}^{t, x} d B_{s}
$$

Thanks to the equivalence of the norms result (3.2), we know that $g\left(X_{T}^{t, x}\right)$ and $f\left(s, X_{s}^{t, x}, 0,0\right)$ make sense in the BSDE (9). Moreover we have

$$
g\left(X_{T}^{t, x}\right) \in \mathbf{L}_{n}^{2}\left(\mathcal{F}_{T}\right) \text { and } f\left(., X^{t, x}, 0,0\right) \in \mathbf{H}_{n}^{2}(0, T) .
$$

It follows from the results from Pardoux [13] that for each $(t, x)$, there exists a unique pair $\left(Y^{t, x}, Z^{t, x}\right) \in \mathbf{S}^{2}(t, T) \times \mathbf{H}_{n \times d}^{2}(t, T)$ of $\left\{\mathcal{F}_{s}^{t}\right\}$ progressively measurable processes, which solves this $\operatorname{BSDE}(g, f)$.

The main result of this section is 
Theorem 4.1. Suppose that assumptions 2.1-2.3 and 2.4 hold. Then there exists a unique weak solution $u \in \mathcal{H}$ of the PDE (2). Moreover we have the probabilistic interpretation of the solution:

$$
u(t, x)=Y_{t}^{t, x}, \quad\left(\sigma^{*} \nabla u\right)(t, x)=Z_{t}^{t, x}, \quad d t \otimes d x-\text { a.e. }
$$

and moreover $Y_{s}^{t, x}=u\left(s, X_{s}^{t, x}\right), Z_{s}^{t, x}=\left(\sigma^{*} \nabla u\right)\left(s, X_{s}^{t, x}\right), d t \otimes d P \otimes d x$-a.e. $\forall s \in[t, T]$.

Proof: We start to prove the existence result.

a) Existence : We prove the existence in three steps. By integration by parts formula, we know that $u$ solves (2) if and only if

$$
\widehat{u}(t, x)=e^{\mu t} u(t, x)
$$

is a solution of the $\operatorname{PDE}(\widehat{g}, \widehat{f})$, where

$$
\widehat{g}(x)=e^{\mu T} g(x) \text { and } \widehat{f}(t, x, y, z)=e^{\mu t} f\left(t, x, e^{-\mu t} y, e^{-\mu t} z\right)-\mu y .
$$

Then the coefficient $\widehat{f}$ satisfies the assumption 2.3 as $f$, except that 2.3 -(iii) is replaced by

$$
\left(y-y^{\prime}\right)\left(f(t, x, y, z)-f\left(t, x, y^{\prime}, z\right)\right) \leqslant 0 .
$$

In the first two steps, we consider the case where $f$ does not depend on $\nabla u$, and write $f(t, x, y)$ for $f(t, x, y, v(t, x))$, where $v$ is in $\mathbf{L}^{2}\left([0, T] \times \mathbb{R}^{d}, d t \otimes \rho(x) d x\right)$.

We assume first that $f(t, x, y)$ satisfies the following assumption 2.3 : $\forall\left(t, x, y, y^{\prime}\right) \in[0, T] \times \mathbb{R}^{d} \times$ $\mathbb{R}^{n} \times \mathbb{R}^{n}$,

(i) $|f(t, x, y)| \leqslant|f(t, x, 0)|+\varphi(|y|)$,

(ii) $\left\langle y-y^{\prime}, f(t, x, y)-f\left(t, x, y^{\prime}\right)\right\rangle \leqslant 0$,

(iii) $y \rightarrow f(t, x, y)$ is continuous, $\forall(t, x) \in[0, T] \times \mathbb{R}^{d}$.

Step 1: Suppose that $g(x), f(t, x, 0)$ are uniformly bounded, i.e. there exists a constant $C$, such that

$$
|g(x)|+\sup _{0 \leqslant t \leqslant T}|f(t, x, 0)| \leqslant C
$$

where $C$ as a constant which can be changed line by line.

Define $f_{n}(t, y):=\left(\theta_{n} * f(t, \cdot)\right)(y)$ where $\theta_{n}: \mathbb{R}^{n} \rightarrow \mathbb{R}_{+}$is a sequence of smooth functions with compact support, which approximate the Dirac distribution at 0 , and satisfy $\int \theta_{n}(z) d z=1$. Let $\left\{\left(Y_{s}^{n, t, x}, Z_{s}^{n, t, x}\right), t \leqslant s \leqslant T\right\}$ be the solution of BSDE associated to $\left(g\left(X_{T}^{t, x}\right), f_{n}\right)$, namely,

$$
Y_{s}^{n, t, x}=g\left(X_{T}^{t, x}\right)+\int_{s}^{T} f\left(r, X_{r}^{t, x}, Y_{r}^{n, t, x}\right) d r-\int_{s}^{T} Z_{r}^{n, t, x} d B_{r}, \text { P-a.s.. }
$$

Then for each $n \in \mathbb{N}$, we have

$$
\left|Y_{s}^{n, t, x}\right| \leqslant e^{T} C
$$


and

$$
\left|f_{n}\left(s, X_{s}^{t, x}, Y_{s}^{n, t, x}\right)\right|^{2} \leqslant 2\left|f_{n}\left(s, X_{s}^{t, x}, 0\right)\right|^{2}+2 \psi^{2}\left(e^{\frac{T}{2} \sqrt{C}}\right)
$$

where $\psi(r):=\sup _{n} \sup _{|y| \leqslant r} \int_{\mathbb{R}^{n}} \varphi(|y|) \theta_{n}(y-z) d z$. So there exists a constant $C>0$, s.t.

$$
\sup _{n} \int_{\mathbb{R}^{d}} E \int_{t}^{T}\left(\left|Y_{s}^{n, t, x}\right|^{2}+\left|f_{n}\left(s, X_{s}^{t, x}, Y_{s}^{n, t, x}\right)\right|^{2}+\left|Z_{s}^{n, t, x}\right|^{2}\right) \rho(x) d s d x \leqslant C .
$$

Then let $n \rightarrow \infty$ on the both sides of (14), we get that the limit $\left(Y_{s}^{t, x}, Z_{s}^{t, x}\right)$ of $\left(Y_{s}^{n, t, x}, Z_{s}^{n, t, x}\right)$, satisfies

$$
Y_{s}^{t, x}=g\left(X_{T}^{t, x}\right)+\int_{s}^{T} f\left(r, X_{r}^{t, x}, Y_{r}^{t, x}\right) d r-\int_{s}^{T} Z_{r}^{t, x} d B_{r}, \text { P-a.s.. }
$$

Moreover we obtain from the estimate (15) that

$$
\int_{\mathbb{R}^{d}} \int_{t}^{T} E\left(\left|Y_{s}^{t, x}\right|^{2}+\left|Z_{s}^{t, x}\right|^{2}\right) \rho(x) d s d x<\infty .
$$

Notice that $\left(Y_{t}^{t, x}, Z_{t}^{t, x}\right)$ are $\mathcal{F}_{t}^{t}$ measurable, which implies they are deterministic. Define $u(t, x):=$ $Y_{t}^{t, x}$, and $v(t, x):=Z_{t}^{t, x}$. By the flow property of $X_{r}^{s, x}$ and by the uniqueness of the solution of the BSDE (16), we have that $Y_{s}^{t, x}=u\left(s, X_{s}^{t, x}\right)$ and $Z_{s}^{t, x}=v\left(s, X_{s}^{t, x}\right)$.

The terminal condition $g$ and $f(., ., 0,0)$ are not continuous in $t$ and $x$, and assumed to belong in a suitable weighted $L^{2}$ space, so the solution $u$ and for instance $v$ are not in general continuous, and are only defined a.e. in $[0, T] \times \mathbb{R}^{d}$. So in order to give meaning to the expression $u\left(s, X_{s}^{t, x}\right)$ (resp. $v\left(s, X_{s}^{t, x}\right)$ ), and following Bally and Matoussi [3], we apply a regularization procedure on the final condition $g$ and the coefficient $f$. Actually, according to Pardoux and Peng ([15], Theorem $3.2)$, if the coefficient $(g, f)$ are smooth, then the PDE (2) admits a unique classical solution $u \in C^{1,2}\left([0, T] \times \mathbb{R}^{d}\right)$. Therefore the approximated expression $u\left(s, X_{s}^{t, x}\right)\left(\operatorname{resp} . v\left(s, X_{s}^{t, x}\right)\right)$ has a meaning and then pass to the limit in $L^{2}$ spaces like us in Bally and Matoussi [3].

Now, the equivalence of norm result (8) and estimate (17) follow that $u, v \in \mathbf{L}^{2}\left([0, T] \times \mathbb{R}^{d}, d t \otimes\right.$ $\rho(x) d x)$. Finally, let $F(r, x)=f\left(r, X_{r}^{t, x}, Y_{r}^{t, x}\right)$, we know that $F(s, x) \in \mathbf{L}^{2}\left([0, T] \times \mathbb{R}^{d}, d t \otimes\right.$ $\rho(x) d x)$, in view of

$$
\begin{aligned}
\int_{\mathbb{R}^{d}} \int_{t}^{T}|F(s, x)|^{2} \rho(x) d s d x & \leqslant \frac{1}{k_{2}} \int_{\mathbb{R}^{d}} \int_{t}^{T} E\left|F\left(s, X_{s}^{t, x}\right)\right|^{2} \rho(x) d s d x \\
& =\frac{1}{k_{2}} \int_{\mathbb{R}^{d}} \int_{t}^{T} E\left|f\left(s, X_{s}^{t, x}, Y_{s}^{t, x}\right)\right|^{2} \rho(x) d s d x<\infty .
\end{aligned}
$$

So that from theorem 2.1 in [3], we get that $v=\sigma^{*} \nabla u$ and that $u \in \mathcal{H}$ solves the PDE associated to $(g, f)$ under the bounded assumption.

Step 2 : We assume $g \in \mathbf{L}^{2}\left(\mathbb{R}^{d}, \rho(x) d x\right), f$ satisfies the assumption 2.3' and $f(t, x, 0) \in$ $\mathbf{L}^{2}\left([0, T] \times \mathbb{R}^{d}, d t \otimes \rho(x) d x\right)$. We approximate $g$ and $f$ by bounded functions as follows :

$$
\begin{aligned}
g_{n}(x) & =\Pi_{n}(g(x)), \\
f_{n}(t, x, y) & =f(t, x, y)-f(t, x, 0)+\Pi_{n}(f(t, x, 0)),
\end{aligned}
$$

where

$$
\Pi_{n}(y):=\frac{\min (n,|y|)}{|y|} y .
$$


Clearly, the pair $\left(g_{n}, f_{n}\right)$ satisfies the assumption (13) of step 1, and

$$
\begin{aligned}
g_{n} & \rightarrow g \text { in } \mathbf{L}^{2}\left(\mathbb{R}^{d}, \rho(x) d x\right), \\
f_{n}(t, x, 0) & \rightarrow f(t, x, 0) \text { in } \mathbf{L}^{2}\left([0, T] \times \mathbb{R}^{d}, d t \otimes \rho(x) d x\right) .
\end{aligned}
$$

Denote $\left(Y_{s}^{n, t, x}, Z_{s}^{n, t, x}\right) \in \mathbf{S}_{n}^{2}(t, T) \times \mathbf{H}_{n \times d}^{2}(t, T)$ the solution of the $\operatorname{BSDE}\left(\xi_{n}, f_{n}\right)$, where $\xi_{n}=$ $g_{n}\left(X_{T}^{t, x}\right)$, i.e.

$$
Y_{s}^{n, t, x}=g_{n}\left(X_{T}^{t, x}\right)+\int_{s}^{T} f_{n}\left(r, X_{r}^{t, x}, Y_{r}^{n, t, x}\right) d r-\int_{s}^{T} Z_{r}^{n, t, x} d B_{r} .
$$

Then from the results in step $1, u_{n}(t, x)=Y_{t}^{n, t, x}$ and $u_{n}(t, x) \in \mathcal{H}$, is the weak solution of the $\operatorname{PDE}\left(g_{n}, f_{n}\right)$, with

$$
Y_{s}^{n, t, x}=u_{n}\left(s, X_{s}^{t, x}\right), Z_{s}^{n, t, x}=\left(\sigma^{*} \nabla u_{n}\right)\left(s, X_{s}^{t, x}\right), \text { a.s. }
$$

For $m, n \in \mathbb{N}$, applying Itô's formula to $\left|Y_{s}^{m, t, x}-Y_{s}^{n, t, x}\right|^{2}$, we get

$$
\begin{aligned}
E\left|Y_{s}^{m, t, x}-Y_{s}^{n, t, x}\right|^{2} & +E \int_{s}^{T}\left|Z_{r}^{m, t, x}-Z_{r}^{n, t, x}\right|^{2} d r \leqslant E\left|g_{m}\left(X_{T}^{t, x}\right)-g_{n}\left(X_{T}^{t, x}\right)\right|^{2} \\
& +E \int_{s}^{T}\left|Y_{r}^{m, t, x}-Y_{r}^{n, t, x}\right|^{2} d r+E \int_{s}^{T}\left|f_{m}\left(r, X_{r}^{t, x}, 0\right)-f_{n}\left(r, X_{r}^{t, x}, 0\right)\right|^{2} d r .
\end{aligned}
$$

From the equivalence of the norms (7) and (8), it follows

$$
\begin{aligned}
& \int_{\mathbb{R}^{d}} E\left|Y_{s}^{m, t, x}-Y_{s}^{n, t, x}\right|^{2} \rho(x) d x \leqslant \int_{\mathbb{R}^{d}} E\left|g_{m}\left(X_{T}^{t, x}\right)-g_{n}\left(X_{T}^{t, x}\right)\right|^{2} \rho(x) d x \\
& \quad+\int_{\mathbb{R}^{d}} E \int_{s}^{T}\left|Y_{r}^{m, t, x}-Y_{r}^{n, t, x}\right|^{2} d r \rho(x) d x+\int_{\mathbb{R}^{d}} E \int_{s}^{T}\left|f_{m}\left(r, X_{r}^{t, x}, 0\right)-f_{n}\left(r, X_{r}^{t, x}, 0\right)\right|^{2} d r \rho(x) d x \\
& \leqslant \int_{\mathbb{R}^{d}} E \int_{s}^{T}\left|Y_{r}^{m, t, x}-Y_{r}^{n, t, x}\right|^{2} d r \rho(x) d x+k_{1} \int_{\mathbb{R}^{d}} E\left|g_{m}(x)-g_{n}(x)\right|^{2} \rho(x) d x \\
& \quad+k_{1} \int_{\mathbb{R}^{d}} \int_{t}^{T}\left|f_{m}(r, x, 0)-f_{n}(r, x, 0)\right|^{2} \rho(x) d r d x,
\end{aligned}
$$

and by Gronwall's inequality and (19), we get as $m, n \rightarrow \infty$

$$
\sup _{t \leqslant s \leqslant T} \int_{\mathbb{R}^{d}} E\left|Y_{s}^{m, t, x}-Y_{s}^{n, t, x}\right|^{2} \rho(x) d x \rightarrow 0 .
$$

It follows immediately as $m, n \rightarrow \infty$

$$
\int_{\mathbb{R}^{d}} E \int_{s}^{T}\left|Y_{r}^{m, t, x}-Y_{r}^{n, t, x}\right|^{2} \rho(x) d r d x+\int_{\mathbb{R}^{d}} E \int_{s}^{T}\left|Z_{r}^{m, t, x}-Z_{r}^{n, t, x}\right|^{2} \rho(x) d r d x \rightarrow 0 .
$$

Using again the equivalence of the norms (8), we get:

$$
\begin{aligned}
& \int_{t}^{T} \int_{\mathbb{R}^{d}}\left|u_{m}(s, x)-u_{n}(s, x)\right|^{2}+\left|\sigma^{*} \nabla u_{m}(s, x)-\sigma^{*} \nabla u_{n}(s, x)\right|^{2} \rho(x) d x d s \\
\leqslant & \frac{1}{k_{2}} \int_{t}^{T} \int_{\mathbb{R}^{d}} E\left(\left|u_{m}\left(s, X_{s}^{t, x}\right)-u_{n}\left(s, X_{s}^{t, x}\right)\right|^{2}+\left|\sigma^{*} \nabla u_{m}\left(s, X_{s}^{t, x}\right)-\sigma^{*} \nabla u_{n}\left(s, X_{s}^{t, x}\right)\right|^{2}\right) \rho(x) d s d x \\
= & \frac{1}{k_{2}} \int_{t}^{T} \int_{\mathbb{R}^{d}} E\left(\left|Y_{s}^{m, t, x}-Y_{s}^{n, t, x}\right|^{2}+\left|Z_{s}^{m, t, x}-Z_{s}^{n, t, x}\right|^{2}\right) \rho(x) d s d x \rightarrow 0 .
\end{aligned}
$$


as $m, n \rightarrow \infty$, i.e. $\left\{u_{n}\right\}$ is Cauchy sequence in $\mathcal{H}$. Denote its limit as $u$, so $u \in \mathcal{H}$, and satisfies for every $\phi \in C_{c}^{1, \infty}\left([0, T] \times \mathbb{R}^{d}\right)$,

$$
\int_{t}^{T}\left(u_{s}, \partial_{t} \phi\right) d s+(u(t, \cdot), \phi(t, \cdot))-(g(\cdot), \phi(\cdot, T))+\int_{t}^{T} \mathcal{E}\left(u_{s}, \phi_{s}\right) d s=\int_{t}^{T}\left(f\left(s, \cdot, u_{s}\right), \phi_{s}\right) d s .
$$

On the other hand, $\left(Y^{n, t, x}, Z_{.}^{n, t, x}\right)$ converges to $\left(Y^{t, x}, Z^{t, x}\right)$ in $\mathbf{S}_{n}^{2}(0, T) \times \mathbf{H}_{n \times d}^{2}(0, T)$, which is the solution of the BSDE with parameters $\left(g\left(X_{T}^{t, x}\right), f\right)$; by the equivalence of the norms, we deduce that

$$
Y_{s}^{t, x}=u\left(s, X_{s}^{t, x}\right), Z_{s}^{t, x}=\sigma^{*} \nabla u\left(s, X_{s}^{t, x}\right), \text { a.s. } \forall s \in[t, T],
$$

specially $Y_{t}^{t, x}=u(t, x), Z_{t}^{t, x}=\sigma^{*} \nabla u(t, x)$.

Now, it's easy to the generalize the result to the case when $f$ satisfies assumption 2.2 .

Step 3: In this step, we consider the case where $f$ depends on $\nabla u$. Assume that $g, f$ satisfy the assumptions $2.1-2.3$, with assumption 2.3-(iii) replaced by (12). From the result in step 2 , for any given $n \times d$-matrix-valued function $v \in \mathbf{L}^{2}\left([0, T] \times \mathbb{R}^{d}, d t \otimes \rho(x) d x\right), f(t, x, u, v(t, x))$ satisfies the assumptions in step 2. So the $\operatorname{PDE}(g, f(t, x, u, v(t, x)))$ admits a unique solution $u \in \mathcal{H}$ satisfying (i) and (ii) in the definition 2.1 .

Set $V_{s}^{t, x}=v\left(s, X_{s}^{t, x}\right)$, then $V_{s}^{t, x} \in \mathbf{H}_{n \times d}^{2}(0, T)$ in view of the equivalence of the norms. We consider the following BSDE with solution $\left(Y^{t, x}, Z^{t, x}\right)$

$$
Y_{s}^{t, x}=g\left(X_{T}^{t, x}\right)+\int_{s}^{T} f\left(s, X_{s}^{t, x}, Y_{s}^{t, x}, V_{s}^{t, x}\right) d s-\int_{s}^{T} Z_{s}^{t, x} d B_{s},
$$

then $Y_{s}^{t, x}=u\left(s, X_{s}^{t, x}\right), Z_{s}^{t, x}=\sigma^{*} \nabla u\left(s, X_{s}^{t, x}\right)$, a.s. $\forall s \in[t, T]$.

Now we can construct a mapping $\Psi$ from $\mathcal{H}$ into itself. For any $\bar{u} \in \mathcal{H}, u=\Psi(\bar{u})$ is the weak solution of the PDE with parameters $g(x)$ and $f\left(t, x, u, \sigma^{*} \nabla \bar{u}\right)$.

Symmetrically we introduce a mapping $\Phi$ from $\mathbf{H}_{n}^{2}(t, T) \times \mathbf{H}_{n \times d}^{2}(t, T)$ into itself. For any $\left(U^{t, x}, V^{t, x}\right) \in \mathbf{H}_{n}^{2}(t, T) \times \mathbf{H}_{n \times d}^{2}(t, T),\left(Y^{t, x}, Z^{t, x}\right)=\Phi\left(U^{t, x}, V^{t, x}\right)$ is the solution of the BSDE with parameters $g\left(X_{T}^{t, x}\right)$ and $f\left(s, X_{s}^{t, x}, Y_{s}^{t, x}, V_{s}^{t, x}\right)$. Set $V_{s}^{t, x}=\sigma^{*} \nabla \bar{u}\left(s, X_{s}^{t, x}\right)$, then $Y_{s}^{t, x}=u\left(s, X_{s}^{t, x}\right)$, $Z_{s}^{t, x}=\sigma^{*} \nabla u\left(s, X_{s}^{t, x}\right)$, a.s.a.e..

Let $\bar{u}_{1}, \bar{u}_{2} \in \mathcal{H}$, and $u_{1}=\Psi\left(\bar{u}_{1}\right), u_{2}=\Psi\left(\bar{u}_{2}\right)$, we consider the difference $\triangle u:=u_{1}-u_{2}$, $\triangle \bar{u}:=\bar{u}_{1}-\bar{u}_{2}$. Set $V_{s}^{t, x, 1}:=\sigma^{*} \nabla \bar{u}_{1}\left(s, X_{s}^{t, x}\right), V_{s}^{t, x, 2}:=\sigma^{*} \nabla \bar{u}_{2}\left(s, X_{s}^{t, x}\right)$. We denote by $\left(Y^{t, x, 1}, Z^{t, x, 1}\right)$ (resp. $\left.\left(Y^{t, x, 2}, Z^{t, x, 2}\right)\right)$ the solution of the BSDE with parameters $g\left(X_{T}^{t, x}\right)$ and $f\left(s, X_{s}^{t, x}, Y_{s}^{t, x}, V_{s}^{t, x, 1}\right)\left(\operatorname{resp} . f\left(s, X_{s}^{t, x}, Y_{s}^{t, x}, V_{s}^{t, x, 2}\right)\right)$; then for a.e. $\forall s \in[t, T]$,

$$
\begin{aligned}
& Y_{s}^{t, x, 1}=u_{1}\left(s, X_{s}^{t, x}\right), Z_{s}^{t, x, 1}=\sigma^{*} \nabla u_{1}\left(s, X_{s}^{t, x}\right), \\
& Y_{s}^{t, x, 2}=u_{2}\left(s, X_{s}^{t, x}\right), Z_{s}^{t, x, 2}=\sigma^{*} \nabla u_{2}\left(s, X_{s}^{t, x}\right),
\end{aligned}
$$

Denote $\triangle Y_{s}^{t, x}:=Y_{s}^{t, x, 1}-Y_{s}^{t, x, 2}, \triangle Z_{s}^{t, x}:=Z_{s}^{t, x, 1}-Z_{s}^{t, x, 2}, \triangle V_{s}^{t, x}:=V_{s}^{t, x, 1}-V_{s}^{t, x, 2}$. By Itô's formula applied to $e^{\gamma t} E\left|\triangle Y_{s}^{t, x}\right|^{2}$, for some $\alpha$ and $\gamma \in \mathbb{R}$, we have

$$
e^{\gamma t} E\left|\triangle Y_{s}^{t, x}\right|^{2}+E \int_{s}^{T} e^{\gamma s}\left(\gamma\left|\triangle Y_{r}^{t, x}\right|^{2}+\left|\triangle Z_{r}^{t, x}\right|^{2}\right) d r \leqslant E \int_{s}^{T} e^{\gamma s}\left(\frac{k^{2}}{\alpha}\left|\triangle Y_{r}^{t, x}\right|^{2}+\alpha\left|\triangle V_{r}^{t, x}\right|^{2}\right) d r,
$$


Using the equivalence of the norms, we deduce that

$$
\begin{aligned}
& \int_{\mathbb{R}^{d}} \int_{t}^{T} e^{\gamma s}\left(\gamma|\triangle u(s, x)|^{2}+\left|\sigma^{*} \nabla(\triangle u)(s, x)\right|^{2}\right) \rho(x) d s d x \\
\leqslant & \frac{1}{k_{2}} \int_{\mathbb{R}^{d}} \int_{t}^{T} e^{\gamma s} E\left(\gamma\left|\triangle Y_{r}^{t, x}\right|^{2}+\left|\triangle Z_{r}^{t, x}\right|^{2}\right) \rho(x) d r d x \\
\leqslant & \frac{1}{k_{2}} \int_{\mathbb{R}^{d}} \int_{s}^{T} e^{\gamma s} E\left(\frac{k^{2}}{\alpha}\left|\triangle Y_{r}^{t, x}\right|^{2}+\alpha\left|\triangle V_{r}^{t, x}\right|^{2}\right) \rho(x) d r d x \\
\leqslant & \frac{k_{1}}{k_{2}} \int_{\mathbb{R}^{d}} \int_{s}^{T} e^{\gamma s}\left(\frac{k^{2}}{\alpha}|\triangle u(s, x)|^{2}+\alpha\left|\sigma^{*} \nabla(\triangle \bar{u})(s, x)\right|^{2}\right) \rho(x) d s d x .
\end{aligned}
$$

Set $\alpha=\frac{k_{2}}{2 k_{1}}, \gamma=1+\frac{2 k_{1}^{2}}{k_{2}^{2}} k^{2}$, then we get

$$
\begin{aligned}
& \int_{\mathbb{R}^{d}} \int_{t}^{T} e^{\gamma s}\left(|\triangle u(s, x)|^{2}+\left|\sigma^{*} \nabla(\triangle u)(s, x)\right|^{2}\right) \rho(x) d s d x \\
\leqslant & \frac{1}{2} \int_{\mathbb{R}^{d}} \int_{t}^{T} e^{\gamma s}\left|\sigma^{*} \nabla(\triangle \bar{u})(s, x)\right|^{2} \rho(x) d s d x \\
\leqslant & \frac{1}{2} \int_{\mathbb{R}^{d}} \int_{t}^{T} e^{\gamma s}\left(|\triangle \bar{u}(s, x)|^{2}+\left|\sigma^{*} \nabla(\triangle \bar{u})(s, x)\right|^{2}\right) \rho(x) d s d x .
\end{aligned}
$$

Consequently, $\Psi$ is a strict contraction on $\mathcal{H}$ equipped with the norm

$$
\|u\|_{\gamma}^{2}:=\int_{\mathbb{R}^{d}} \int_{t}^{T} e^{\gamma s}\left(|u(s, x)|^{2}+\left|\sigma^{*} \nabla u(s, x)\right|^{2}\right) \rho(x) d s d x .
$$

So $\Psi$ has fixed point $u \in \mathcal{H}$ which is the solution of the PDE (2) associated to $(g, f)$. Moreover, for $t \leqslant s \leqslant T$,

$$
Y_{s}^{t, x}=u\left(s, X_{s}^{t, x}\right), Z_{s}^{t, x}=\sigma^{*} \nabla u\left(s, X_{s}^{t, x}\right), \text {.a.e. }
$$

and specially $Y_{t}^{t, x}=u(t, x), Z_{t}^{t, x}=\sigma^{*} \nabla u(t, x)$, a.e.

b) Uniqueness : Let $u^{1}$ and $u^{2} \in \mathcal{H}$ be two solutions of the $\operatorname{PDE}(g, f)$. From Proposition 3.1, for $\phi \in C_{c}^{2}\left(\mathbb{R}^{d}\right)$ and $i=1,2$

$$
\begin{aligned}
& \int_{\mathbb{R}^{d}} \int_{s}^{T} u^{i}(r, x) d \phi_{t}(r, x) d x+\left(u^{i}(s, \cdot), \phi_{t}(s, \cdot)\right)-\left(g(\cdot), \phi_{t}(\cdot, T)\right)-\int_{s}^{T} \mathcal{E}\left(u^{i}(r, \cdot), \phi_{t}(r, \cdot)\right) d r \\
= & \int_{s}^{T} \int_{\mathbb{R}^{d}} \phi_{t}(r, x) f\left(r, x, u^{i}(r, x), \sigma^{*} \nabla u^{i}(r, x)\right) d r d x .
\end{aligned}
$$

By (4), we get

$$
\begin{aligned}
\int_{\mathbb{R}^{d}} \int_{s}^{T} u^{i} d \phi_{t}(r, x) d x= & \int_{s}^{T}\left(\int_{\mathbb{R}^{d}}\left(\sigma^{*} \nabla u^{i}\right)(r, x) \phi_{t}(r, x) d x\right) d B_{r} \\
& +\int_{s}^{T} \int_{\mathbb{R}^{d}}\left(\left(\sigma^{*} \nabla u^{i}\right)\left(\sigma^{*} \nabla \phi_{r}\right)+\phi \nabla\left(\left(\frac{1}{2} \sigma^{*} \nabla \sigma+b\right) u_{r}^{i}\right)\right) d x d r .
\end{aligned}
$$


We substitute this in (23), and get

$$
\begin{aligned}
\int_{\mathbb{R}^{d}} u^{i}(s, x) \phi_{t}(s, x) d x= & \left(g(\cdot), \phi_{t}(\cdot, T)\right)-\int_{s}^{T} \int_{\mathbb{R}^{d}}\left(\sigma^{*} \nabla u^{i}\right)(r, x) \phi_{t}(r, x) d x d B_{r} \\
& +\int_{s}^{T} \int_{\mathbb{R}^{d}} \phi_{t}(r, x) f\left(r, x, u^{i}(r, x), \sigma^{*} \nabla u^{i}(r, x)\right) d r d x .
\end{aligned}
$$

Then by the change of variable $y=\widehat{X}_{r}^{t, x}$, we obtain

$$
\begin{aligned}
\int_{\mathbb{R}^{d}} u^{i}\left(s, X_{s}^{t, y}\right) \phi(y) d y= & \int_{\mathbb{R}^{d}} g\left(X_{T}^{t, y}\right) \phi(y) d y+\int_{s}^{T} \int_{\mathbb{R}^{d}} \phi(y) f\left(s, X_{s}^{t, y}, u^{i}\left(s, X_{s}^{t, y}\right), \sigma^{*} \nabla u^{i}\left(s, X_{s}^{t, y}\right)\right) d y d s \\
& -\int_{s}^{T} \int_{\mathbb{R}^{d}}\left(\sigma^{*} \nabla u^{i}\right)\left(r, X_{r}^{t, y}\right) \phi(y) d y d B_{r} .
\end{aligned}
$$

Since $\phi$ is arbitrary, we can prove this result for $\rho(y) d y$ almost every $y$. So $\left(u^{i}\left(s, X_{s}^{t, y}\right),\left(\sigma^{*} \nabla u^{i}\right)\left(s, X_{s}^{t, y}\right)\right)$ solves the $\operatorname{BSDE}\left(g\left(X_{T}^{t, y}\right), f\right)$, i.e. $\rho(y) d y$ a.s., we have

$$
u^{i}\left(s, X_{s}^{t, y}\right)=g\left(X_{T}^{t, y}\right)+\int_{s}^{T} f\left(s, X_{s}^{t, y}, u^{i}\left(s, X_{s}^{t, y}\right), \sigma^{*} \nabla u^{i}\left(s, X_{s}^{t, y}\right)\right) d s-\int_{s}^{T}\left(\sigma^{*} \nabla u^{i}\right)\left(r, X_{r}^{t, y}\right) d B_{r} .
$$

Then by the uniqueness of the BSDE, we know $u^{1}\left(s, X_{s}^{t, y}\right)=u^{2}\left(s, X_{s}^{t, x}\right)$ and $\left(\sigma^{*} \nabla u^{1}\right)\left(s, X_{s}^{t, y}\right)=$ $\left(\sigma^{*} \nabla u^{2}\right)\left(s, X_{s}^{t, y}\right)$. Taking $s=t$ we deduce that $u^{1}(t, y)=u^{2}(t, y), d t \otimes d y$-a.s.

\section{Sobolev's solution for PDE with obstacle under monotonicity condition}

In this section we study the PDE with obstacle associated with $(g, f, h)$, which satisfy the assumptions 2.1-2.4 for $n=1$. We will prove the existence and uniqueness of a weak solution to the obstacle problem. We will restrict our study to the case when $\varphi$ is polynomial increasing in $y$, i.e.

Assumption 5.1. We assume that for some $\kappa_{1} \in \mathbb{R}, \beta_{1}>0, \forall y \in \mathbb{R}$,

$$
|\varphi(y)| \leqslant \kappa_{1}\left(1+|y|^{\beta_{1}}\right) \text {. }
$$

For the sake of PDE with obstacle, we introduce the reflected BSDE associated with $(g, f, h)$, like in El Karoui et al. [7]:

$$
\left\{\begin{array}{l}
Y_{s}^{t, x}=g\left(X_{T}^{t, x}\right)+\int_{s}^{T} f\left(r, X_{r}^{t, x}, Y_{r}^{t, x}, Z_{r}^{t, x}\right) d r+K_{T}^{t, x}-K_{t}^{t, x}-\int_{s}^{T} Z_{s}^{t, x} d B_{s}, P-a . s \forall s \in[t, T] \\
Y_{s}^{t, x} \geqslant L_{s}^{t, x}, P-a . s \\
\int_{t}^{T}\left(Y_{s}^{t, x}-L_{s}^{t, x}\right) d K_{s}^{t, x}=0, P \text {-a.s. }
\end{array}\right.
$$


where $L_{s}^{t, x}=h\left(s, X_{s}^{t, x}\right)$ is a continuous process. Moreover following Lepeltier et al [12], we shall need to estimate

$$
\begin{aligned}
E\left[\sup _{t \leqslant s \leqslant T} \varphi^{2}\left(e^{\mu t}\left(L_{s}^{t, x}\right)^{+}\right)\right] & =E\left[\sup _{t \leqslant s \leqslant T} \varphi^{2}\left(e^{\mu t} h\left(s, X_{s}^{t, x}\right)^{+}\right)\right] \\
& \leqslant C e^{2 \beta_{1} \mu T} E\left[\sup _{t \leqslant s \leqslant T}\left(1+\left|X_{s}^{t, x}\right|^{2 \beta_{1} \beta}\right)\right] \\
& \leqslant C\left(1+|x|^{2 \beta_{1} \beta}\right),
\end{aligned}
$$

where $C$ is a constant which can be changed line by line. By assumption 2.4-(ii), with same techniques we get for $x \in \mathbb{R}, E\left[\sup _{t \leqslant s \leqslant T} \varphi^{2}\left(\left(L_{s}^{t, x}\right)^{+}\right)\right]<+\infty$. Thanks to the assumption 2.1 and 2.2 , by the equivalence of norms 7 and 8 , we have

$$
g\left(X_{T}^{t, x}\right) \in \mathbf{L}^{2}\left(\mathcal{F}_{T}\right) \text { and } f\left(s, X_{s}^{t, x}, 0,0\right) \in \mathbf{H}^{2}(0, T) .
$$

By the existence and uniqueness theorem for the RBSDE in [12], for each $(t, x)$, there exists a unique triple $\left(Y^{t, x}, Z^{t, x}, K^{t, x}\right) \in \mathbf{S}^{2}(t, T) \times \mathbf{H}_{d}^{2}(t, T) \times \mathbf{A}^{2}(t, T)$ of $\left\{\mathcal{F}_{s}^{t}\right\}$ progressively measurable processes, which is the solution of the reflected BSDE with parameters $\left(g\left(X_{T}^{t, x}\right), f\left(s, X_{s}^{t, x}, y, z\right)\right.$, $\left.h\left(s, X_{s}^{t, x}\right)\right)$ We shall give the probabilistic interpretation for the solution of PDE with obstacle $(3)$.

The main result of this section is

Theorem 5.1. Assume that assumptions 2.1-2.5 hold and $\rho(x)=(1+|x|)^{-p}$ with $p \geqslant \gamma$ where $\gamma=\beta_{1} \beta+\beta+d+1$. There exists a pair $(u, \nu)$, which is the solution of the PDE with obstacle (3) associated to $(g, f, h)$ i.e. $(u, \nu)$ satisfies Definition 2.2-(i) -(iii). Moreover the solution is given by: $u(t, x)=Y_{t}^{t, x}$, a.e. where $\left(Y_{s}^{t, x}, Z_{s}^{t, x}, K_{s}^{t, x}\right)_{t \leqslant s \leqslant T}$ is the solution of RBSDE (24), and

$$
Y_{s}^{t, x}=u\left(s, X_{s}^{t, x}\right), Z_{s}^{t, x}=\left(\sigma^{*} \nabla u\right)\left(s, X_{s}^{t, x}\right) .
$$

Moreover, we have for every measurable bounded and positive functions $\phi$ and $\psi$,

$$
\int_{\mathbb{R}^{d}} \int_{t}^{T} \phi\left(s, \widehat{X}_{s}^{t, x}\right) J\left(\widehat{X}_{s}^{t, x}\right) \psi(s, x) 1_{\{u=h\}}(s, x) d \nu(s, x)=\int_{\mathbb{R}^{d}} \int_{t}^{T} \phi(s, x) \psi\left(s, X_{s}^{t, x}\right) d K_{s}^{t, x}, \text { a.s.. }
$$

If $(\bar{u}, \bar{\nu})$ is another solution of the PDE (3) such that $\bar{\nu}$ satisfies (26) with some $\bar{K}$ instead of $K$, where $\bar{K}$ is a continuous process in $\mathbf{A}_{\mathcal{F}}^{2}(t, T)$, then $\bar{u}=u$ and $\bar{\nu}=\nu$.

Remark 5.1. The expression (26) gives us the probabilistic interpretation (Feymamn-Kac's formula) for the measure $\nu$ via the increasing process $K^{t, x}$ of the RBSDE. This formula was first introduced in Bally et al. [1], where the authors prove (26) when $f$ is Lipschitz on $y$ and $z$ uniformly in $(t, \omega)$. Here we generalize their result to the case when $f$ is monotonic in $y$ and Lipschitz in $z$.

Proof. As in the proof of theorem 4.1 in section 4, we first notice that $(u, \nu)$ solves (3) if and only if

$$
(\widehat{u}(t, x), d \widehat{\nu}(t, x))=\left(e^{\mu t} u(t, x), e^{\mu t} d \nu(t, x)\right)
$$

is the solution of the PDE with obstacle $(\widehat{g}, \widehat{f}, \widehat{h})$, where $\widehat{g}, \widehat{f}$ are defined as in (12) with

$$
\widehat{h}(t, x)=e^{\mu t} h(t, x) .
$$


Then the coefficient $\widehat{f}$ satisfies the same assumptions in assumption 2.3 with (iii) replaced by (12), which means that $f$ is decreasing on $y$ in the 1 -dimensional case. The obstacle $\widehat{h}$ still satisfies assumption 2.4 , for $\mu=0$. In the following we will use $(g, f, h)$ instead of $(\widehat{g}, \widehat{f}, \widehat{h})$, and suppose that $(g, f, h)$ satisfies assumption $2.1,2.2,2.4,2.5$ and 2.3 with (iii) replaced by (12).

a) Existence : The existence of a solution will be proved in 4 steps. From step 1 to step 3, we suppose that $f$ does not depend on $\nabla u$, satisfies assumption [2.3' for $n=1$, and $f(t, x, 0) \in$ $\mathbf{L}^{2}\left([0, T] \times \mathbb{R}^{d}, d t \otimes \rho(x) d x\right)$. In the step 4 , we study the case when $f$ depend on $\nabla u$.

Step 1 : Suppose $g(x), f(t, x, 0), h^{+}(t, x)$ uniformly bounded i.e. that there exists a constant $C$ such that

$$
|g(x)|+\sup _{0 \leqslant t \leqslant T}|f(t, x, 0)|+\sup _{0 \leqslant t \leqslant T} h^{+}(t, x) \leqslant C .
$$

We will use the penalization method. For $n \in \mathbb{N}$, we consider for all $s \in[t, T]$,

$$
Y_{s}^{n, t, x}=g\left(X_{T}^{t, x}\right)+\int_{s}^{T} f\left(r, X_{r}^{t, x}, Y_{r}^{n, t, x}\right) d r+n \int_{s}^{T}\left(Y_{r}^{n, t, x}-h\left(r, X_{r}^{t, x}\right)\right)^{-} d r-\int_{s}^{T} Z_{r}^{n, t, x} d B_{r} .
$$

From Theorem 4.1 in section 3 , we know that $u_{n}(t, x):=Y_{t}^{n, t, x}$, is solution of the $\operatorname{PDE}\left(g, f_{n}\right)$, where $f_{n}(t, x, y, x)=f(t, x, y, z)+n(y-h(t, x))^{-}$, i.e. for every $\phi \in C_{c}^{1, \infty}\left([0, T] \times \mathbb{R}^{d}\right)$

$$
\begin{aligned}
\int_{t}^{T}\left(u_{s}^{n}, \partial_{s} \phi\right) d s & +\left(u^{n}(t, \cdot), \phi(t, \cdot)\right)-(g(\cdot), \phi(\cdot, T))+\int_{t}^{T} \mathcal{E}\left(u_{s}^{n}, \phi_{s}\right) d s \\
& =\int_{t}^{T}\left(f\left(s, \cdot, u_{s}^{n}\right), \phi_{s}\right) d s+n \int_{t}^{T}\left(\left(u^{n}-h\right)^{-}(s, \cdot), \phi_{s}\right) d s .
\end{aligned}
$$

Moreover

$$
Y_{s}^{n, t, x}=u_{n}\left(s, X_{s}^{t, x}\right), Z_{s}^{n, t, x}=\sigma^{*} \nabla u_{n}\left(s, X_{s}^{t, x}\right),
$$

Set $K_{s}^{n, t, x}=n \int_{t}^{s}\left(Y_{r}^{n, t, x}-h\left(r, X_{r}^{t, x}\right)\right)^{-} d r$. Then by (27), we have that $K_{s}^{n, t, x}=n \int_{t}^{s}\left(u_{n}-\right.$ $h)^{-}\left(r, X_{r}^{t, x}\right) d r$.

Following the estimates and convergence results for $\left(Y^{n, t, x}, Z^{n, t, x}\right)$ in the step 1 of the proof of Theorem 2.2 in [12], for $m, n \in \mathbb{N}$, we have, as $m, n \rightarrow \infty$

$$
E \int_{t}^{T}\left|Y_{s}^{n, t, x}-Y_{s}^{m, t, x}\right|^{2} d s+E \int_{t}^{T}\left|Z_{s}^{n, t, x}-Z_{s}^{m, t, x}\right|^{2} d s+E \sup _{t \leqslant s \leqslant T}\left|K_{s}^{n, t, x}-K_{s}^{m, t, x}\right|^{2} \rightarrow 0,
$$

and

$$
\sup _{n} E \int_{0}^{T}\left(\left|Y_{s}^{n, t, x}\right|^{2}+\left|Z_{s}^{n, t, x}\right|^{2}+\left(K_{T}^{n, t, x}\right)^{2}\right) \leqslant C .
$$

By the equivalence of the norms (8), we get

$$
\begin{aligned}
& \int_{\mathbb{R}^{d}} \int_{t}^{T} \rho(x)\left(\left|u_{n}(s, x)-u_{m}(s, x)\right|^{2}+\left|\sigma^{*} \nabla u_{n}(s, x)-\sigma^{*} \nabla u_{m}(s, x)\right|^{2}\right) d s d x \\
\leqslant & \frac{1}{k_{2}} \int_{\mathbb{R}^{d}} \rho(x) E \int_{t}^{T}\left(\left|Y_{s}^{n, t, x}-Y_{s}^{m, t, x}\right|^{2}+\left|Z_{s}^{n, t, x}-Z_{s}^{m, t, x}\right|^{2}\right) d s d x \rightarrow 0 .
\end{aligned}
$$

Thus $\left(u_{n}\right)$ is a Cauchy sequence in $\mathcal{H}$, and the limit $u=\lim _{n \rightarrow \infty} u_{n}$ belongs to $\mathcal{H}$. 
Denote $\nu_{n}(d t, d x)=n\left(u_{n}-h\right)^{-}(t, x) d t d x$ and $\pi_{n}(d t, d x)=\rho(x) \nu_{n}(d t, d x)$, then by (7)

$$
\begin{aligned}
\pi_{n}\left([0, T] \times \mathbb{R}^{d}\right) & =\int_{\mathbb{R}^{d}} \int_{0}^{T} \rho(x) \nu_{n}(d t, d x)=\int_{\mathbb{R}^{d}} \int_{0}^{T} \rho(x) n\left(u_{n}-h\right)^{-}(t, x) d t d x \\
& \leqslant \frac{1}{k_{2}} \int_{\mathbb{R}^{d}} \rho(x) E\left|K_{T}^{n, 0, x}\right| d x \leqslant C \int_{\mathbb{R}^{d}} \rho(x) d x<\infty .
\end{aligned}
$$

It follows that

$$
\sup _{n} \pi_{n}\left([0, T] \times \mathbb{R}^{d}\right)<\infty .
$$

In the same way like in the existence proof step 2 of theorem 14 in [1], we can prove that $\pi_{n}\left([0, T] \times \mathbb{R}^{d}\right)$ is bounded and then $\pi_{n}$ is tight. So we may pass to a subsequence and get $\pi_{n} \rightarrow \pi$ where $\pi$ is a positive measure. Define $\nu=\rho^{-1} \pi ; \nu$ is a positive measure such that $\int_{0}^{T} \int_{\mathbb{R}^{d}} \rho(x) d \nu(t, x)<\infty$, and so we have for $\phi \in C_{c}^{1, \infty}\left([0, T] \times \mathbb{R}^{d}\right)$ with compact support in $x$,

$$
\int_{\mathbb{R}^{d}} \int_{t}^{T} \phi d \nu_{n}=\int_{\mathbb{R}^{d}} \int_{t}^{T} \frac{\phi}{\rho} d \pi_{n} \rightarrow \int_{\mathbb{R}^{d}} \int_{t}^{T} \frac{\phi}{\rho} d \pi=\int_{\mathbb{R}^{d}} \int_{t}^{T} \phi d \nu .
$$

Now passing to the limit in the $\operatorname{PDE}\left(g, f_{n}\right)$, we check that $(u, \nu)$ satisfies the PDE with obstacle $(g, f, h)$, i.e. for every $\phi \in C_{c}^{1, \infty}\left([0, T] \times \mathbb{R}^{d}\right)$, we have

$$
\begin{aligned}
& \int_{t}^{T}\left(u_{s}, \partial_{s} \phi\right) d s+(u(t, \cdot), \phi(t, \cdot))-(g(\cdot), \phi(\cdot, T))+\int_{t}^{T} \mathcal{E}\left(u_{s}, \phi_{s}\right) d s \\
= & \int_{t}^{T}\left(f\left(s, \cdot, u_{s}\right), \phi_{s}\right) d s+\int_{t}^{T} \int_{\mathbb{R}^{d}} \phi(s, x) 1_{\{u=h\}}(s, x) d \nu(x, s) .
\end{aligned}
$$

The last is to prove that $\nu$ satisfies the probabilistic interpretation (26). Since $K^{n, t, x}$ converges to $K^{t, x}$ uniformly in $t$, the measure $d K^{n, t, x} \rightarrow d K^{t, x}$ weakly in probability.

Fix two continuous functions $\phi, \psi:[0, T] \times \mathbb{R}^{d} \rightarrow \mathbb{R}^{+}$which have compact support in $x$ and a continuous function with compact support $\theta: \mathbb{R}^{d} \rightarrow \mathbb{R}^{+}$, we have

$$
\begin{aligned}
& \int_{\mathbb{R}^{d}} \int_{t}^{T} \phi\left(s, \widehat{X}_{s}^{t, x}\right) J\left(\widehat{X}_{s}^{t, x}\right) \psi(s, x) \theta(x) d \nu(s, x) \\
= & \lim _{n \rightarrow \infty} \int_{\mathbb{R}^{d}} \int_{t}^{T} \phi\left(s, \widehat{X}_{s}^{t, x}\right) J\left(\widehat{X}_{s}^{t, x}\right) \psi(s, x) \theta(x) n\left(u_{n}-h\right)^{-}(t, x) d t d x \\
= & \lim _{n \rightarrow \infty} \int_{\mathbb{R}^{d}} \int_{t}^{T} \phi(s, x) \psi\left(s, X_{s}^{t, x}\right) \theta\left(X_{s}^{t, x}\right) n\left(u_{n}-h\right)^{-}\left(t, X_{s}^{t, x}\right) d t d x \\
= & \lim _{n \rightarrow \infty} \int_{\mathbb{R}^{d}} \int_{t}^{T} \phi(s, x) \psi\left(s, X_{s}^{t, x}\right) \theta\left(X_{s}^{t, x}\right) d K_{s}^{n, t, x} d x \\
= & \int_{\mathbb{R}^{d}} \int_{t}^{T} \phi(s, x) \psi\left(s, X_{s}^{t, x}\right) \theta\left(X_{s}^{t, x}\right) d K_{s}^{t, x} d x .
\end{aligned}
$$

We take $\theta=\theta_{R}$ to be the regularization of the indicator function of the ball of radius $R$ and pass to the limit with $R \rightarrow \infty$, it follows that

$$
\int_{\mathbb{R}^{d}} \int_{t}^{T} \phi\left(s, \widehat{X}_{s}^{t, x}\right) J\left(\widehat{X}_{s}^{t, x}\right) \psi(s, x) d \nu(s, x)=\int_{\mathbb{R}^{d}} \int_{t}^{T} \phi(s, x) \psi\left(s, X_{s}^{t, x}\right) d K_{s}^{t, x} d x .
$$


Since $\left(Y_{s}^{n, t, x}, Z_{s}^{n, t, x}, K_{s}^{n, t, x}\right)$ converges to $\left(Y_{s}^{t, x}, Z_{s}^{t, x}, K_{s}^{t, x}\right)$ as $n \rightarrow \infty$ in $\mathbf{S}^{2}(t, T) \times \mathbf{H}^{2}(t, T) \times$ $\mathbf{A}^{2}(t, T)$, and $\left(Y_{s}^{t, x}, Z_{s}^{t, x}, K_{s}^{t, x}\right)$ is the solution of $\operatorname{RBSDE}\left(g\left(X_{T}^{t, x}\right), f, h\right)$, then we have

$$
\int_{t}^{T}\left(Y_{s}^{t, x}-L_{s}^{t, x}\right) d K_{s}^{t, x}=\int_{t}^{T}(u-h)\left(t, X_{s}^{t, x}\right) d K_{s}^{t, x}=0, \text { a.s. }
$$

it follows that $d K_{s}^{t, x}=1_{\{u=h\}}\left(s, X_{s}^{t, x}\right) d K_{s}^{t, x}$. In (30), setting $\psi=1_{\{u=h\}}$ yields

$$
\int_{\mathbb{R}^{d}} \int_{t}^{T} \phi\left(s, \widehat{X}_{s}^{t, x}\right) J\left(\widehat{X}_{s}^{t, x}\right) 1_{\{u=h\}}(s, x) d \nu(s, x)=\int_{\mathbb{R}^{d}} \int_{t}^{T} \phi\left(s, \widehat{X}_{s}^{t, x}\right) J\left(\widehat{X}_{s}^{t, x}\right) d \nu(s, x), \text { a.s. }
$$

Note that the family of functions $A(\omega)=\left\{(s, x) \rightarrow \phi\left(s, \widehat{X}_{s}^{t, x}\right): \phi \in C_{c}^{\infty}\right\}$ is an algebra which separates the points (because $x \rightarrow \widehat{X}_{s}^{t, x}$ is a bijection). Given a compact set $G, A(\omega)$ is dense in $C([0, T] \times G)$. It follows that $J\left(\widehat{X}_{s}^{t, x}\right) 1_{\{u=h\}}(s, x) d \nu(s, x)=J\left(\widehat{X}_{s}^{t, x}\right) d \nu(s, x)$ for almost every $\omega$. While $J\left(\widehat{X}_{s}^{t, x}\right)>0$ for almost every $\omega$, we get $d \nu(s, x)=1_{\{u=h\}}(s, x) d \nu(s, x)$, and (26) follows.

Then we get easily that $Y_{s}^{t, x}=u\left(s, X_{s}^{t, x}\right)$ and $Z_{s}^{t, x}=\sigma^{*} \nabla u\left(s, X_{s}^{t, x}\right)$, in view of the convergence results for $\left(Y_{s}^{n, t, x}, Z_{s}^{n, t, x}\right)$ and the equivalence of the norms. So $u\left(s, X_{s}^{t, x}\right)=Y_{s}^{t, x} \geqslant h(t, x)$. Specially for $s=t$, we have $u(t, x) \geqslant h(t, x)$

Step 2: As in the proof of the RBSDE in Theorem 2.2 in [12], step 2, we relax the bounded condition on the barrier $h$ in step 1 , and prove the existence of the solution under assumption 2.4.

Similarly to step 2 in the proof of theorem 2.2 in [12], after some transformation, we know that it is sufficient to prove the existence of the solution for the PDE with obstacle $(g, f, h)$, where $(g, f, h)$ satisfies

$$
g(x), f(t, x, 0) \leqslant 0
$$

Let $h(t, x)$ satisfy assumption 2.4 for $\mu=0$, i.e. $\forall(t, x) \in[0, T] \times, \mathbb{R}^{d}$

$$
\varphi\left(h(t, x)^{+}\right) \in \mathbf{L}^{2}\left(\mathbb{R}^{d} ; \rho(x) d x\right),
$$

and

$$
|h(t, x)| \leqslant \kappa\left(1+|x|^{\beta}\right) .
$$

Set

$$
h_{n}(t, x)=h(t, x) \wedge n,
$$

then the function $h_{n}(t, x)$ are continuous, $\sup _{0} \leqslant t \leqslant T h_{n}^{+}(t, x) \leqslant n$, and $h_{n}\left(s, X_{s}^{t, x}\right) \rightarrow h\left(s, X_{s}^{t, x}\right)$ in $\mathbf{S}_{\mathcal{F}}^{2}(t, T)$, in view of Dini's theorem and dominated convergence theorem.

We consider the PDE with obstacle associated with $\left(g, f, h_{n}\right)$. By the results of step 1 , there exists $\left(u_{n}, \nu_{n}\right)$, which is the solution of the PDE with obstacle associated to $\left(g, f, h_{n}\right)$, where $u_{n} \in \mathcal{H}$ and $\nu_{n}$ is a positive measure such that $\int_{0}^{T} \int_{\mathbb{R}^{d}} \rho(x) d \nu_{n}(t, x)<\infty$. Moreover

$$
\left\{\begin{array}{l}
Y_{s}^{n, t, x}=u_{n}\left(s, X_{s}^{t, x}\right), Z_{s}^{n, t, x}=\sigma^{*} \nabla u_{n}\left(s, X_{s}^{t, x}\right), \\
\int_{\mathbb{R}^{d}} \int_{t}^{T} \phi\left(s, \widehat{X}_{s}^{t, x}\right) J\left(\widehat{X}_{s}^{t, x}\right) \psi(s, x) 1_{\left\{u_{n}=h_{n}\right\}}(s, x) d \nu_{n}(s, x)=\int_{\mathbb{R}^{d}} \int_{t}^{T} \phi(s, x) \psi\left(s, X_{s}^{t, x}\right) d K_{s}^{n, t, x} d x,
\end{array}\right.
$$


Here $\left(Y^{n, t, x}, Z^{n, t, x}, K^{n, t, x}\right)$ is the solution of the $\operatorname{RBSDE}\left(g\left(X_{T}^{t, x}\right), f, h_{n}\right)$. Thanks to proposition 6.1 in Appendix, and the bounded assumption of $g$ and $f$, we know that

$$
\begin{aligned}
& E\left[\int_{t}^{T}\left(\left|Y_{s}^{n, t, x}\right|^{2}+\left|Z_{s}^{n, t, x}\right|^{2}\right) d s+\left(K_{T}^{n, 0, x}\right)^{2}\right] \\
& \leqslant C\left(1+E\left[\varphi^{2}\left(\sup _{0 \leqslant t \leqslant T} h^{+}\left(t, X_{t}^{0, x}\right)\right)+\sup _{0 \leqslant t \leqslant T}\left(h^{+}\left(t, X_{t}^{0, x}\right)\right)^{2}\right]\right) \\
& \leqslant C\left(1+|x|^{2 \beta_{1} \beta}+|x|^{2 \beta}\right) .
\end{aligned}
$$

By the Lemma 2.3 in [12], $Y_{s}^{n, t, x} \rightarrow Y_{s}^{t, x}$ in $\mathbf{S}^{2}(0, T), Z_{s}^{n, t, x} \rightarrow Z_{s}^{t, x}$ in $\mathbf{H}_{d}^{2}(0, T)$ and $K_{s}^{n, t, x} \rightarrow K_{s}^{t, x}$ in $\mathbf{A}^{2}(0, T)$, as $n \rightarrow \infty$. Moreover $\left(Y_{s}^{t, x}, Z_{s}^{t, x}, K_{s}^{t, x}\right)$ is the solution of $\operatorname{RBSDE}\left(g\left(X_{T}^{t, x}\right), f, h\right)$.

By the convergence result of $\left(Y_{s}^{n, t, x}, Z_{s}^{n, t, x}\right)$ and the equivalence of the norms (8), we get

$$
\begin{aligned}
& \int_{\mathbb{R}^{d}} \rho(x) \int_{t}^{T}\left(\left|u_{n}(t, x)-u_{m}(t, x)\right|^{2}+\left|\sigma^{*} \nabla u_{n}(s, x)-\sigma^{*} \nabla u_{m}(s, x)\right|^{2}\right) d s d x \\
\leqslant & \frac{1}{k_{2}} \int_{\mathbb{R}^{d}} \rho(x) E \int_{t}^{T}\left(\left|Y_{s}^{n, t, x}-Y_{s}^{m, t, x}\right|^{2}+\left|Z_{s}^{n, t, x}-Z_{s}^{m, t, x}\right|^{2}\right) d s d x \rightarrow 0 .
\end{aligned}
$$

So $\left\{u_{n}\right\}$ is a Cauchy sequence in $\mathcal{H}$, and admits a limit $u \in \mathcal{H}$. Moreover $Y_{s}^{t, x}=u\left(s, X_{s}^{t, x}\right), Z_{s}^{t, x}=$ $\sigma^{*} \nabla u\left(s, X_{s}^{t, x}\right)$. In particular $u(t, x)=Y_{t}^{t, x} \geqslant h(t, x)$.

Set $\pi_{n}=\rho \nu_{n}$, like in step 1 , we first need to prove that $\pi_{n}\left([0, T] \times \mathbb{R}^{d}\right)$ is uniformly bounded. In (31), let $\phi=\rho, \psi=1$, then we have

$$
\int_{\mathbb{R}^{d}} \int_{0}^{T} \rho\left(\widehat{X}_{s}^{0, x}\right) J\left(\widehat{X}_{s}^{0, x}\right) d \nu_{n}(s, x)=\int_{\mathbb{R}^{d}} \int_{0}^{T} \rho(x) d K_{s}^{n, 0, x} d x .
$$

Recall Lemma 3.2 ; there exist two constants $c_{1}>0$ and $c_{2}>0$ such that $\forall x \in \mathbb{R}^{d}, 0 \leqslant t \leqslant T$

$$
c_{1} \leqslant E\left(\frac{\rho\left(t, \widehat{X}_{t}^{0, x}\right) J\left(\widehat{X}_{t}^{0, x}\right)}{\rho(x)}\right) \leqslant c_{2} .
$$


Applying Hölder's inequality and Schwartz's inequality, we have

$$
\begin{aligned}
& \pi_{n}\left([0, T] \times \mathbb{R}^{d}\right) \\
= & \int_{\mathbb{R}^{d}} \int_{0}^{T} \rho(x) \nu_{n}(d t, d x) \\
= & \int_{\mathbb{R}^{d}} \int_{0}^{T} \frac{\rho^{\frac{1}{2}}(x)}{\rho^{\frac{1}{2}}\left(t, \widehat{X}_{t}^{0, x}\right) J^{\frac{1}{2}}\left(\widehat{X}_{t}^{0, x}\right)} \rho^{\frac{1}{2}}(x) \rho^{\frac{1}{2}}\left(t, \widehat{X}_{t}^{0, x}\right) J^{\frac{1}{2}}\left(\widehat{X}_{t}^{0, x}\right) \nu_{n}(d t, d x) \\
\leqslant & E\left[\left(\int_{\mathbb{R}^{d}} \int_{0}^{T} \frac{\rho(x)}{\rho\left(t, \widehat{X}_{t}^{0, x}\right) J\left(\widehat{X}_{t}^{0, x}\right)} \rho(x) \nu_{n}(d t, d x)\right)^{\frac{1}{2}}\left(\int_{\mathbb{R}^{d}} \int_{0}^{T} \rho\left(t, \widehat{X}_{t}^{0, x}\right) J\left(\widehat{X}_{t}^{0, x}\right) \nu_{n}(d t, d x)\right)^{\frac{1}{2}}\right] \\
\leqslant & \left(E \int_{\mathbb{R}^{d}} \int_{0}^{T} \frac{\rho(x)}{\rho\left(t, \widehat{X}_{t}^{0, x}\right) J\left(\widehat{X}_{t}^{0, x}\right)} \rho(x) \nu_{n}(d t, d x)\right)^{\frac{1}{2}}\left(E \int_{\mathbb{R}^{d}} \int_{0}^{T} \rho\left(t, \widehat{X}_{t}^{0, x}\right) J\left(\widehat{X}_{t}^{0, x}\right) \nu_{n}(d t, d x)\right)^{\frac{1}{2}} \\
= & \left(\int_{\mathbb{R}^{d}} \int_{0}^{T} E\left(\frac{\rho(x)}{\rho\left(t, \widehat{X}_{t}^{0, x}\right) J\left(\widehat{X}_{t}^{0, x}\right)}\right) \rho(x) \nu_{n}(d t, d x)\right)^{\frac{1}{2}}\left(\int_{\mathbb{R}^{d}} E \int_{0}^{T} d K_{t}^{n, 0, x} \rho(x) d x\right)^{\frac{1}{2}} \\
\leqslant & \left(\frac{1}{c_{1}} \int_{\mathbb{R}^{d}} \int_{0}^{T} \rho(x) \nu_{n}(d t, d x)\right)^{\frac{1}{2}}\left(\int_{\mathbb{R}^{d}} \rho(x) E\left[K_{T}^{n, 0, x}\right] d x\right)^{\frac{1}{2}} \cdot
\end{aligned}
$$

So by (32) and (7), we get

$$
\begin{aligned}
\sup _{n} \pi_{n}\left([0, T] \times \mathbb{R}^{d}\right) & \leqslant C \int_{\mathbb{R}^{d}} \rho(x) E\left[K_{T}^{n, 0, x}\right] d x \\
& \leqslant C \int_{\mathbb{R}^{d}} \rho(x)\left(1+|x|^{\beta_{1} \beta}+|x|^{\beta}\right) d x<\infty .
\end{aligned}
$$

Using the same arguments as in step 1, we deduce that $\pi_{n}$ is tight. So we may pass to a subsequence and get $\pi_{n} \rightarrow \pi$ where $\pi$ is a positive measure.

Define $\nu=\rho^{-1} \pi$, then $\nu$ is a positive measure such that $\int_{0}^{T} \int_{\mathbb{R}^{d}} \rho(x) d \nu(t, x)<\infty$. Then for $\phi \in C\left([0, T] \times \mathbb{R}^{d}\right)$ with compact support in $x$, we have as $n \rightarrow \infty$,

$$
\int_{t}^{T} \int \phi d \nu_{n}=\int_{t}^{T} \int \frac{\phi}{\rho} d \pi_{n} \rightarrow \int_{t}^{T} \int \frac{\phi}{\rho} d \pi=\int_{t}^{T} \int \phi d \nu .
$$

Now passing to the limit in the $\operatorname{PDE}\left(g, f, h_{n}\right)$, we check that $(u, \nu)$ satisfies the PDE with obstacle associated to $(g, f, h)$, i.e. for every $\phi \in C_{c}^{1, \infty}\left([0, T] \times \mathbb{R}^{d}\right)$

$$
\begin{aligned}
& \int_{t}^{T}\left(u_{s}, \partial_{s} \phi\right) d s+(u(t, \cdot), \phi(t, \cdot))-(g(\cdot), \phi(\cdot, T))+\int_{t}^{T} \mathcal{E}\left(u_{s}, \phi_{s}\right) d s \\
= & \int_{t}^{T}\left(f\left(s, \cdot, u_{s}\right), \phi_{s}\right) d s+\int_{t}^{T} \int_{\mathbb{R}^{d}} \phi(s, x) 1_{\{u=h\}} d \nu(x, s) .
\end{aligned}
$$

Then we will check if the probabilistic interpretation (26) still holds. Fix two continuous functions $\phi, \psi:[0, T] \times \mathbb{R}^{d} \rightarrow \mathbb{R}^{+}$which have compact support in $x$. With the convergence result of 
$K^{n, t, x}$, which implies $d K^{n, t, x} \rightarrow d K^{t, x}$ weakly in probability, in the same way as step 1, passing to the limit in (31) we have

$$
\int_{\mathbb{R}^{d}} \int_{t}^{T} \phi\left(s, \widehat{X}_{s}^{t, x}\right) J\left(\widehat{X}_{s}^{t, x}\right) \psi(s, x) d \nu(s, x)=\int_{\mathbb{R}^{d}} \int_{t}^{T} \phi(s, x) \psi\left(s, X_{s}^{t, x}\right) d K_{s}^{t, x} d x
$$

Since $\left(Y_{s}^{t, x}, Z_{s}^{t, x}, K_{s}^{t, x}\right)$ is the solution of $\operatorname{RBSDE}\left(g\left(X_{T}^{t, x}\right), f, h\right)$, then by the integral condition, we deduce the $d K_{s}^{t, x}=1_{\{u=h\}}\left(s, X_{s}^{t, x}\right) d K_{s}^{t, x}$. In (35), setting $\psi=1_{\{u=h\}}$ yields

$$
\int_{\mathbb{R}^{d}} \int_{t}^{T} \phi\left(s, \widehat{X}_{s}^{t, x}\right) J\left(\widehat{X}_{s}^{t, x}\right) 1_{\{u=h\}}(s, x) d \nu(s, x)=\int_{\mathbb{R}^{d}} \int_{t}^{T} \phi\left(s, \widehat{X}_{s}^{t, x}\right) J\left(\widehat{X}_{s}^{t, x}\right) d \nu(s, x) .
$$

With the same arguments, we get that $d \nu(s, x)=1_{\{u=h\}}(s, x) d \nu(s, x)$, and (26) holds for $\nu$ and $K$.

Step 3: Now we will relax the bounded condition on $g(x)$ and $f(t, x, 0)$. Then for $m, n \in \mathbb{N}$, let

$$
\begin{aligned}
g_{m, n}(x) & =(g(x) \wedge n) \vee(-m), \\
f_{m, n}(t, x, y) & =f(t, x, y)-f(t, x, 0)+(f(t, x, 0) \wedge n) \vee(-m) .
\end{aligned}
$$

So $g_{m, n}(x)$ and $f_{m, n}(t, x, 0)$ are bounded and for fixed $m \in \mathbb{N}$, as $n \rightarrow \infty$, we have

$$
\begin{aligned}
g_{m, n}(x) & \rightarrow g_{m}(x) \text { in } \mathbf{L}^{2}\left(\mathbb{R}^{d}, \rho(x) d x\right), \\
f_{m, n}(t, x, 0) & \rightarrow f_{m}(t, x, 0) \text { in } \mathbf{L}^{2}\left([0, T] \times \mathbb{R}^{d}, d t \otimes \rho(x) d x\right),
\end{aligned}
$$

where

$$
\begin{aligned}
g_{m}(x) & =g(x) \vee(-m), \\
f_{m}(t, x, y) & =f(t, x, y)-f(t, x, 0)+f(t, x, 0) \vee(-m) .
\end{aligned}
$$

Then as $m \rightarrow \infty$, we have

$$
\begin{aligned}
g_{m}(x) & \rightarrow g(x) \text { in } \mathbf{L}^{2}\left(\mathbb{R}^{d}, \rho(x) d x\right), \\
f_{m}(t, x, 0) & \rightarrow f(t, x, 0) \text { in } \mathbf{L}^{2}\left([0, T] \times \mathbb{R}^{d}, d t \otimes \rho(x) d x\right),
\end{aligned}
$$

in view of assumption 2.1 and $f(t, x, 0) \in \mathbf{L}^{2}\left([0, T] \times \mathbb{R}^{d}, d t \otimes \rho(x) d x\right)$.

Now we consider the PDE with obstacle associated to $\left(g_{m, n}, f_{m, n}, h\right)$. By step 2, there exists a $\left(u_{m, n}, \nu_{m, n}\right)$ which is the solution of the PDE with obstacle associated to $\left(g_{m, n}, f_{m, n}, h\right)$. In particular the representation formulas (25) and (26) are satisfied. Denote by $\left(Y^{m, n, t, x}, Z^{m, n, t, x}, K^{m, n, t, x}\right)$ the solution of the RBSDE $\left(g_{m, n}\left(X_{T}^{t, x}\right), f_{m, n}, h\right)$.

Recall the convergence results in step 3 of theorem 2.2 in [12], we know that for fixed $m \in \mathbb{N}$, as $n \rightarrow \infty,\left(Y_{s}^{m, n, t, x}, Z_{s}^{m, n, t, x}, K_{s}^{m, n, t, x}\right) \rightarrow\left(Y_{s}^{m, t, x}, Z_{s}^{m, t, x}, K_{s}^{m, t, x}\right)$ in $\mathbf{S}^{2}(0, T) \times \mathbf{H}_{d}^{2}(0, T) \times \mathbf{A}^{2}(0, T)$, and that $\left(Y_{s}^{m, t, x}, Z_{s}^{m, t, x}, K_{s}^{m, t, x}\right)$ is the solution of $\operatorname{RBSDE}\left(g_{m}\left(X_{T}^{t, x}\right), f_{m}, h\right)$.

By Itô's formula, we have for $n, p \in \mathbb{N}$,

$$
\begin{aligned}
& E \int_{t}^{T}\left(\left|Y_{s}^{m, n, t, x}-Y_{s}^{m, p, t, x}\right|^{2}+\left|Z_{s}^{m, n, t, x}-Z_{s}^{m, p, t, x}\right|^{2}\right) d s \\
\leqslant & C E\left|g_{m, n}\left(X_{T}^{t, x}\right)-g_{m, p}\left(X_{T}^{t, x}\right)\right|^{2}+C E \int_{t}^{T}\left|f_{m, n}\left(s, X_{s}^{t, x}, 0\right)-f_{m, p}\left(s, X_{s}^{t, x}, 0\right)\right|^{2} d s,
\end{aligned}
$$


so by the equivalence of the norms (7) and (8), it follows that as $n \rightarrow \infty$,

$$
\begin{aligned}
& \int_{\mathbb{R}^{d}} \int_{t}^{T} \rho(x)\left(\left|u_{m, n}(t, x)-u_{m, p}(t, x)\right|^{2}+\left|\sigma^{*} \nabla u_{m, n}(s, x)-\sigma^{*} \nabla u_{m, p}(s, x)\right|^{2}\right) d s d x \\
\leqslant & \frac{C k_{1}}{k_{2}} \int_{\mathbb{R}^{d}} \rho(x)\left|g_{m, n}(x)-g_{m, p}(x)\right|^{2} d x+\frac{C k_{1}}{k_{2}} \int_{\mathbb{R}^{d}} \int_{t}^{T} \rho(x)\left|f_{m, n}(s, x, 0)-f_{m, p}(s, x, 0)\right|^{2} d s d x \rightarrow 0 .
\end{aligned}
$$

i.e. for each fixed $m \in \mathbb{N},\left\{u_{m, n}\right\}$ is a Cauchy sequence in $\mathcal{H}$, and admits a limit $u_{m} \in$ $\mathcal{H}$. Moreover $Y_{s}^{m, t, x}=u_{m}\left(s, X_{s}^{t, x}\right), Z_{s}^{m, t, x}=\sigma^{*} \nabla u_{m}\left(s, X_{s}^{t, x}\right)$, a.s., in particular $u_{m}(t, x)=$ $Y_{t}^{m, t, x} \geqslant h(t, x)$.

Then we find the measure $\nu_{m}$ by the sequence $\left\{\nu_{m, n}\right\}$. Set $\pi_{m, n}=\rho \nu_{m, n}$, by proposition 6.1 in Appendix, we have for each $m, n \in \mathbb{N}, 0 \leqslant t \leqslant T$

$$
\begin{aligned}
E\left(\left|K_{T}^{m, n, t, x}\right|^{2}\right) \leqslant & C E\left[g_{m, n}^{2}\left(X_{T}^{t, x}\right)+\int_{0}^{T} f_{m, n}^{2}\left(s, X_{s}^{t, x}, 0,0\right) d s+\varphi^{2}\left(\sup _{t \leqslant s \leqslant T}\left(h^{+}\left(s, X_{s}^{t, x}\right)\right)\right)\right. \\
& \left.+\sup _{t \leqslant s \leqslant T}\left(h^{+}\left(s, X_{s}^{t, x}\right)\right)^{2}+1+\varphi^{2}(2 T)\right] \\
\leqslant & C E\left[g\left(X_{T}^{t, x}\right)^{2}+\int_{0}^{T} f^{2}\left(s, X_{s}^{t, x}, 0,0\right) d s+\varphi^{2}\left(\sup _{0 \leqslant s \leqslant T}\left(h^{+}\left(s, X_{s}^{t, x}\right)\right)\right)\right. \\
& \left.+\sup _{0 \leqslant s \leqslant T}\left(h^{+}\left(s, X_{s}^{t, x}\right)\right)^{2}+1+\varphi^{2}(2 T)\right] \\
\leqslant & C\left(1+|x|^{\beta_{1} \beta}+|x|^{2 \beta}\right) .
\end{aligned}
$$

By the same way as in step 2 , we deduce that for each fixed $m \in \mathbb{N}, \pi_{m, n}$ is tight, we may pass to a subsequence and get $\pi_{m, n} \rightarrow \pi_{m}$ where $\pi_{m}$ is a positive measure. If we define $\nu_{m}=\rho^{-1} \pi_{m}$, then $\nu_{m}$ is a positive measure such that $\int_{0}^{T} \int_{\mathbb{R}^{d}} \rho(x) d \nu_{m}(t, x)<\infty$. So we have for all $\phi \in C\left([0, T] \times \mathbb{R}^{d}\right)$ with compact support in $x$,

$$
\int_{t}^{T} \int \phi d \nu_{m, n}=\int_{t}^{T} \int \frac{\phi}{\rho} d \pi_{m, n} \rightarrow \int_{t}^{T} \int \frac{\phi}{\rho} d \pi_{m}=\int_{t}^{T} \int \phi d \nu_{m} .
$$

Now for each fixed $m \in \mathbb{N}$, let $n \rightarrow \infty$, in the $\operatorname{PDE}\left(g_{m, n}, f_{m, n}, h\right)$, we check that $\left(u_{m}, \nu_{m}\right)$ satisfies the PDE with obstacle associated to $\left(g_{m}, f_{m}, h\right)$, and by the weak convergence result of $d K^{m, n, t, x}$, we have easily that the probabilistic interpretation (26) holds for $\nu_{m}$ and $K^{m}$.

Then let $m \rightarrow \infty$, by the convergence results in step 4 of theorem 2.2 in [12], we apply the same method as before. We deduce that $\lim _{m \rightarrow \infty} u_{m}=u$ is in $\mathcal{H}$ and $Y_{s}^{t, x}=u\left(s, X_{s}^{t, x}\right), Z_{s}^{t, x}=$ $\sigma^{*} \nabla u\left(s, X_{s}^{t, x}\right)$, a.s., where $\left(Y^{t, x}, Z^{t, x}, K^{t, x}\right)$ is the solution of the $\operatorname{RBSDE}(g, f, h)$, in particular, setting $s=t, u(t, x)=Y_{t}^{t, x} \geqslant h(t, x)$.

From (35), it follows that

$$
E\left[\left(K_{T}^{m, t, x}\right)^{2}\right] \leqslant C\left(1+|x|^{2 \beta \beta_{1}}+|x|^{2 \beta}\right) .
$$

By the same arguments, we can find the measure $\nu$ by the sequence $\left\{\nu_{m}\right\}$, which satisfies that for all $\phi$ and $\psi$ with compact support,

$$
\int_{\mathbb{R}^{d}} \int_{t}^{T} \phi\left(s, \widehat{X}_{s}^{t, x}\right) J\left(\widehat{X}_{s}^{t, x}\right) \psi(s, x) 1_{\{u=h\}}(s, x) d \nu(s, x)=\int_{\mathbb{R}^{d}} \int_{t}^{T} \phi(s, x) \psi\left(s, X_{s}^{t, x}\right) d K_{s}^{t, x} d x .
$$


Finally we find a solution $(u, \nu)$ to the PDE with obstacle $(g, f, h)$, when $f$ does not depend on $\nabla u$. So for every $\phi \in C_{c}^{1, \infty}\left([0, T] \times \mathbb{R}^{d}\right)$

$$
\begin{aligned}
& \int_{t}^{T}\left(u_{s}, \partial_{s} \phi\right) d s+(u(t, \cdot), \phi(t, \cdot))-(g(\cdot), \phi(\cdot, T))+\int_{t}^{T} \mathcal{E}\left(u_{s}, \phi_{s}\right) d s \\
= & \int_{t}^{T}\left(f\left(s, \cdot, u_{s}\right), \phi_{s}\right) d s+\int_{t}^{T} \int_{\mathbb{R}^{d}} \phi(s, x) 1_{\{u=h\}} d \nu(x, s) .
\end{aligned}
$$

Step 4 : Finally we study the case when $f$ depends on $\nabla u$, and satisfies a Lipschitz condition on $\nabla u$. We construct a mapping $\Psi$ from $\mathcal{H}$ into itself. For some $\bar{u} \in \mathcal{H}$, define

$$
u=\Psi(\bar{u})
$$

where $(u, \nu)$ is a weak solution of the PDE with obstacle $(g, f(t, x, u, \sigma \nabla \bar{u}), h)$. Then by this mapping, we denote a sequence $\left\{u_{n}\right\}$ in $\mathcal{H}$, beginning with a function $v^{0} \in \mathbf{L}^{2}\left([0, T] \times \mathbb{R}^{d}, d t \otimes \rho(x) d x\right)$. Since $f\left(t, x, u, v^{0}(t, x)\right)$ satisfies the assumptions of step 3 , the $\operatorname{PDE}\left(g, f\left(t, x, u, v^{0}(t, x)\right), h\right)$ admits a solution $\left(u_{1}, v_{1}\right) \in \mathcal{H}$. For $n \in \mathbb{N}$, set $u_{n}(t, x)=\Psi\left(u_{n-1}(t, x)\right)$.

Symmetrically we introduce a mapping $\Phi$ from $\mathbf{H}^{2}(t, T) \times \mathbf{H}_{d}^{2}(t, T)$ into itself. For $V^{t, x, 0}=$ $\left.v^{0}\left(s, X_{s}^{t, x}\right)\right)$, then $V_{s}^{t, x} \in \mathbf{H}_{d}^{2}(t, T)$ in view of the equivalence of the norms. Set

$$
\left(Y^{t, x, n}, Z^{t, x, n}\right)=\Phi\left(Y^{t, x, n-1}, Z^{t, x, n-1}\right),
$$

where $\left(Y^{t, x, n}, Z^{t, x, n}, K^{t, x, n}\right)$ is the solution of the RBSDE with parameters $g\left(X_{T}^{t, x}\right)$, $f\left(s, X_{s}^{t, x}, Y_{s}^{t, x}, Z_{s}^{t, x, n-1}\right)$ and $h\left(s, X_{s}^{t, x}\right)$. Then $Y_{s}^{t, x, n}=u_{n}\left(s, X_{s}^{t, x}\right), Z_{s}^{t, x, n}=\sigma^{*} \nabla u_{n}\left(s, X_{s}^{t, x}\right)$ a.s. and

$$
\int_{\mathbb{R}^{d}} \int_{t}^{T} \phi\left(s, \widehat{X}_{s}^{t, x}\right) J\left(\widehat{X}_{s}^{t, x}\right) \psi(s, x) 1_{\{u=h\}}(s, x) d \nu_{n}(s, x)=\int_{\mathbb{R}^{d}} \int_{t}^{T} \phi(s, x) \psi\left(s, X_{s}^{t, x}\right) d K_{s}^{t, x, n} d x .
$$

Set $\widetilde{u}_{n}(t, x):=u_{n}(t, x)-u_{n-1}(t, x)$. To deal with the difference $\widetilde{u}_{n}$, we need the difference of the corresponding BSDE, denote $\tilde{Y}_{s}^{t, x, n}:=Y_{s}^{t, x, n}-Y_{s}^{t, x, n-1}, \widetilde{Z}_{s}^{t, x, n}:=Z_{s}^{t, x, n}-Z_{s}^{t, x, n-1}$, $\widetilde{K}_{s}^{t, x, n}:=K_{s}^{t, x, n}-K_{s}^{t, x, n-1}$. It follows from Itô's formula, for some $\alpha, \gamma \in \mathbb{R}$,

$$
\begin{aligned}
e^{\gamma t} E\left|\widetilde{Y}_{s}^{t, x, n}\right|^{2} & +E \int_{s}^{T} e^{\gamma r}\left(\gamma\left|\widetilde{Y}_{r}^{t, x, n}\right|^{2}+\left|\widetilde{Z}_{r}^{t, x, n}\right|^{2}\right) d r \\
& \leqslant E \int_{s}^{T} e^{\gamma r}\left(\frac{k^{2}}{\alpha}\left|\widetilde{Y}_{r}^{t, x, n}\right|^{2}+\alpha\left|\widetilde{Z}_{r}^{t, x, n-1}\right|^{2}\right) d r
\end{aligned}
$$

since

$$
\begin{aligned}
& \int_{s}^{T} e^{\gamma r} \widetilde{Y}_{r}^{t, x, n} d \widetilde{K}_{r}^{t, x, n} \\
= & \int_{s}^{T} e^{\gamma r}\left(Y_{s}^{t, x, n}-h\left(r, X_{r}^{t, x}\right)\right) d K^{t, x, n}+\int_{s}^{T} e^{\gamma r}\left(Y_{s}^{t, x, n-1}-h\left(r, X_{r}^{t, x}\right)\right) d K^{t, x, n-1} \\
& \quad-\int_{s}^{T} e^{\gamma r}\left(Y_{s}^{t, x, n}-h\left(r, X_{r}^{t, x}\right)\right) d K^{t, x, n-1}+\int_{s}^{T} e^{\gamma r}\left(Y_{s}^{t, x, n-1}-h\left(r, X_{r}^{t, x}\right)\right) d K^{t, x, n} \\
\leqslant & 0 .
\end{aligned}
$$


then by the equivalence of the norms, for $\gamma=1+\frac{2 k_{1}^{2}}{k_{2}^{2}} k^{2}$, we have

$$
\begin{aligned}
& \int_{\mathbb{R}^{d}} \int_{t}^{T} e^{\gamma s}\left(\left|\widetilde{u}_{n}(s, x)\right|^{2}+\left|\sigma^{*} \nabla\left(\widetilde{u}_{n}\right)(s, x)\right|^{2}\right) \rho(x) d s d x \\
\leqslant & \left(\frac{1}{2}\right)^{n-1} \int_{\mathbb{R}^{d}} \int_{t}^{T} e^{\gamma s}\left(\left|\widetilde{u}_{2}(s, x)\right|^{2}+\left|\sigma^{*} \nabla\left(\widetilde{u}_{2}\right)(s, x)\right|^{2}\right) \rho(x) d s d x \\
\leqslant & \left(\frac{1}{2}\right)^{n-1}\left(\left\|u_{1}(s, x)\right\|_{\gamma}^{2}+\left\|u_{2}(s, x)\right\|_{\gamma}^{2}\right) .
\end{aligned}
$$

where $\|u\|_{\gamma}^{2}:=\int_{\mathbb{R}^{d}} \int_{t}^{T} e^{\gamma s}\left(|u(s, x)|^{2}+\left|\sigma^{*} \nabla u(s, x)\right|^{2}\right) \rho(x) d s d x$, which is equivalent to the norm $\|\cdot\|$ of $\mathcal{H}$. So $\left\{u_{n}\right\}$ is a Cauchy sequence in $\mathcal{H}$, it admits a limit $u$ in $\mathcal{H}$, which is the solution to the PDE with obstacle (2). Then consider $\sigma^{*} \nabla u$ as a known function by the result of step 3, we know that there exists a positive measure $\nu$ such that $\int_{0}^{T} \int_{\mathbb{R}^{d}} \rho(x) d \nu(t, x)<\infty$, and for every $\phi \in C_{c}^{1, \infty}\left([0, T] \times \mathbb{R}^{d}\right)$,

$$
\begin{aligned}
& \int_{t}^{T}\left(u_{s}, \partial_{s} \phi\right) d s+(u(t, \cdot), \phi(t, \cdot))-(g(\cdot), \phi(\cdot, T))+\int_{t}^{T} \mathcal{E}\left(u_{s}, \phi_{s}\right) d s \\
= & \int_{t}^{T}\left(f\left(s, \cdot, u_{s}, \sigma^{*} \nabla u_{s}\right), \phi_{s}\right) d s+\int_{t}^{T} \int_{\mathbb{R}^{d}} \phi(s, x) 1_{\{u=h\}} d \nu(x, s) .
\end{aligned}
$$

Moreover, for $t \leqslant s \leqslant T$,

$$
Y_{s}^{t, x}=u\left(s, X_{s}^{t, x}\right), Z_{s}^{t, x}=\sigma^{*} \nabla u\left(s, X_{s}^{t, x}\right), \text { a.s.a.e., }
$$

and

$$
\begin{aligned}
& \int_{\mathbb{R}^{d}} \int_{t}^{T} \phi\left(s, \widehat{X}_{s}^{t, x}\right) J\left(\widehat{X}_{s}^{t, x}\right) \psi(s, x) 1_{\{u=h\}}(s, x) d \nu(s, x) \\
= & \int_{\mathbb{R}^{d}} \int_{t}^{T} \phi(s, x) \psi\left(s, X_{s}^{t, x}\right) d K_{s}^{t, x} .
\end{aligned}
$$

b) Uniqueness : Set $(\bar{u}, \bar{\nu})$ to be another solution of the PDE with obstacle (3) associated to $(g, f, h)$; with $\bar{\nu}$ verifies (26) for an increasing process $\bar{K}$. We fix $\phi: \mathbb{R}^{d} \rightarrow \mathbb{R}$, a smooth function in $C_{c}^{2}\left(\mathbb{R}^{d}\right)$ with compact support and denote $\phi_{t}(s, x)=\phi\left(\widehat{X}_{s}^{t, x}\right) J\left(\widehat{X}_{s}^{t, x}\right)$. From proposition 3.1, one may use $\phi_{t}(s, x)$ as a test function in the $\operatorname{PDE}(g, f, h)$ with $\partial_{s} \phi(s, x) d s$ replaced by a stochastic integral with respect to the semimartingale $\phi_{t}(s, x)$. Then we get, for $t \leqslant s \leqslant T$

$$
\begin{aligned}
& \int_{\mathbb{R}^{d}} \int_{s}^{T} \bar{u}(r, x) d \phi_{t}(r, x) d x+\left(\bar{u}(s, \cdot), \phi_{t}(s, \cdot)\right)-\left(g(\cdot), \phi_{t}(\cdot, T)\right)+\int_{s}^{T} \mathcal{E}\left(\bar{u}_{r}, \phi_{r}\right) d r \\
= & \int_{s}^{T} \int_{\mathbb{R}^{d}} f\left(r, x, \bar{u}(r, x), \sigma^{*} \nabla \bar{u}(r, x)\right) \phi_{t}(r, \cdot) d r+\int_{s}^{T} \int_{\mathbf{R}^{d}} \phi_{t}(r, x) 1_{\{\bar{u}=h\}} d \bar{\nu}(x, r) .
\end{aligned}
$$

By (5) in Lemma 3.1, we have

$$
\begin{aligned}
\int_{\mathbb{R}^{d}} \int_{s}^{T} \bar{u} d_{r} \phi_{t}(r, x) d x= & \int_{s}^{T}\left(\int_{\mathbb{R}^{d}}\left(\sigma^{*} \nabla \bar{u}\right)(r, x) \phi_{t}(r, x) d x\right) d B_{r} \\
& +\int_{s}^{T} \int_{\mathbb{R}^{d}}\left(\left(\sigma^{*} \nabla \bar{u}\right)\left(\sigma^{*} \nabla \phi_{r}\right)+\phi_{t} \nabla\left(\left(\frac{1}{2} \sigma^{*} \nabla \sigma+b\right) \bar{u}\right)\right) d x d r .
\end{aligned}
$$


Substitute this equality in (38), we get

$$
\begin{aligned}
\int_{\mathbb{R}^{d}} \bar{u}(s, x) \phi_{t}(s, x) d x= & \left(g(\cdot), \phi_{t}(\cdot, T)\right)-\int_{s}^{T}\left(\int_{\mathbb{R}^{d}}\left(\sigma^{*} \nabla \bar{u}\right)(r, x) \phi_{t}(r, x) d x\right) d B_{r} \\
& +\int_{\mathbb{R}^{d}} \int_{s}^{T} f\left(r, x, \bar{u}(r, x), \sigma^{*} \nabla \bar{u}(r, x)\right) \phi_{t}(s, \cdot) d r+\int_{s}^{T} \int_{\mathbf{R}^{d}} \phi_{t}(r, x) 1_{\{\bar{u}=h\}} d \bar{\nu}(x, r) .
\end{aligned}
$$

Then by changing of variable $y=\widehat{X}_{r}^{t, x}$ and applying (26) for $\bar{\nu}$, we obtain

$$
\begin{aligned}
& \int_{\mathbb{R}^{d}} \bar{u}\left(s, X_{s}^{t, y}\right) \phi(y) d y \\
= & \int_{\mathbb{R}^{d}} g\left(X_{T}^{t, y}\right) \phi(y) d y+\int_{s}^{T} \phi(y) f\left(s, X_{s}^{t, y}, \bar{u}\left(s, X_{s}^{t, y}\right), \sigma^{*} \nabla \bar{u}\left(s, X_{s}^{t, y}\right) d s\right. \\
& +\int_{s}^{T} \int_{\mathbb{R}^{d}} \phi(y) 1_{\{\bar{u}=h\}}\left(r, X_{s}^{t, y}\right) d \bar{K}_{r}^{t, y} d y-\int_{s}^{T}\left(\int_{\mathbb{R}^{d}}\left(\sigma^{*} \nabla \bar{u}\right)\left(r, X_{r}^{t, y}\right) \phi(y) d y\right) d B_{r} .
\end{aligned}
$$

Since $\phi$ is arbitrary, we can prove that for $\rho(y) d y$ almost every $y$, $\left(\bar{u}\left(s, X_{s}^{t, y}\right),\left(\sigma^{*} \nabla \bar{u}\right)\left(s, X_{s}^{t, y}\right), \widehat{K}_{s}^{t, x}\right) \quad$ solves $\quad$ the $\operatorname{RBSDE}\left(g\left(X_{T}^{t, y}\right), f, h\right)$. Here $\widehat{K}_{s}^{t, x}=$ $\int_{t}^{s} 1_{\{\bar{u}=h\}}\left(r, X_{r}^{t, y}\right) d \bar{K}_{r}^{t, y}$. Then by the uniqueness of the solution of the reflected BSDE, we know $\bar{u}\left(s, X_{s}^{t, y}\right)=Y_{s}^{t, y}=u\left(s, X_{s}^{t, x}\right),\left(\sigma^{*} \nabla \bar{u}\right)\left(s, X_{s}^{t, y}\right)=Z_{s}^{t, y}=\left(\sigma^{*} \nabla u\right)\left(s, X_{s}^{t, y}\right)$ and $\widehat{K}_{s}^{t, y}=K_{s}^{t, y}$. Taking $s=t$ we deduce that $\bar{u}(t, y)=u(t, y), \rho(y) d y$-a.s. and by the probabilistic interpretation (26), we obtain

$$
\int_{s}^{T} \int \phi_{t}(r, x) 1_{\{\bar{u}=h\}}(r, x) d \bar{\nu}(x, r)=\int_{s}^{T} \int \phi_{t}(r, x) 1_{\{u=h\}}(r, x) d \nu(x, r) .
$$

So $1_{\{\bar{u}=h\}}(r, x) d \bar{\nu}(x, r)=1_{\{u=h\}}(r, x) d \nu(x, r)$.

\section{Appendix}

\subsection{Proof of proposition 3.1}

First we consider the case when $f$ does not depend on $z$ and satisfies assumption 2.3'. As in step 2 of the proof of theorem 4.1, we approximate $g$ and $f$ as in (18), then $g_{n} \rightarrow g$ in $\mathbf{L}^{2}\left(\mathbb{R}^{d}, \rho(x) d x\right)$ and $f_{n}(t, x, 0) \rightarrow f(t, x, 0)$ in $\mathbf{L}^{2}\left([0, T] \times \mathbb{R}^{d}, d t \otimes \rho(x) d x\right)$, as $n \rightarrow \infty$.

Since for each $n \in \mathbb{N},\left|g_{n}\right| \leqslant n$ and $\left|f_{n}(t, x, 0)\right| \leqslant n$, by the result of the step 1 of theorem 4.1, the $\operatorname{PDE}\left(g_{n}, f_{n}\right)$ admits the weak solution $u_{n} \in \mathcal{H}$ and $\sup _{0} \leqslant t \leqslant T\left|u_{n}(t, x)\right| \leqslant C_{n}$. So we know

$$
\left|f_{n}\left(t, x, u_{n}(t, x)\right)\right|^{2} \leqslant\left|f_{n}(t, x, 0)\right|^{2}+\varphi\left(\sup _{0 \leqslant t \leqslant T}\left|u_{n}(t, x)\right|\right) \leqslant C_{n} .
$$

Set $F_{n}(t, x):=f_{n}\left(t, x, u_{n}(t, x)\right)$, then $F_{n}(t, x) \in \mathbf{L}^{2}\left([0, T] \times \mathbb{R}^{n}, d t \otimes \rho(x) d x\right)$.

From proposition 2.3 in Bally and Matoussi [3], for $\phi \in C_{c}^{2}\left(\mathbb{R}^{d}\right)$, we get, for $t \leqslant s \leqslant T$

$$
\begin{aligned}
& \int_{\mathbb{R}^{d}} \int_{s}^{T} u_{n}(r, x) d \phi_{t}(r, x) d x+\left(u_{n}(s, \cdot), \phi_{t}(s, \cdot)\right)-\left(g_{n}(\cdot), \phi_{t}(\cdot, T)\right)+\int_{s}^{T} \mathcal{E}\left(u_{n}(r, \cdot), \phi_{t}(r, \cdot)\right) d r \\
& =\int_{\mathbb{R}^{d}} \int_{s}^{T} f\left(r, x, u_{n}(r, x)\right) \phi_{t}(r, x) d r d x+\int_{\mathbb{R}^{d}} \int_{s}^{T}\left(f_{n}(r, x, 0)-f(r, x, 0)\right) \phi_{t}(r, x) d r d x .
\end{aligned}
$$


By step 2, we know that as $n \rightarrow \infty, u_{n} \rightarrow u$ in $\mathcal{H}$, where $u$ is a weak solution of the $\operatorname{PDE}(g, f)$, i.e.

$$
\begin{aligned}
u_{n} & \rightarrow u \text { in } \mathbf{L}^{2}\left([0, T] \times \mathbb{R}^{d}, d t \otimes \rho(x) d x\right), \\
\sigma^{*} \nabla u_{n} & \rightarrow \nabla u \text { in } \mathbf{L}^{2}\left([0, T] \times \mathbb{R}^{d}, d t \otimes \rho(x) d x\right) .
\end{aligned}
$$

Then there exists a function $u^{*}$ in $\mathbf{L}^{2}\left([0, T] \times \mathbb{R}^{d}, d t \otimes \rho(x) d x\right)$, such that for a subsequence of $\left\{u_{n}\right\},\left|u_{n_{k}}\right| \leqslant\left|u^{*}\right|$ and $u_{n_{k}} \rightarrow u, d t \otimes d x$-a.e. Thanks to assumption 2.3'-(iii), we have that $f\left(r, x, u_{n}(r, x)\right) \rightarrow f(r, x, u(r, x)), d t \otimes d x$-a.e. Now, for all compact support function $\phi \in C_{c}^{2}\left(\mathbb{R}^{d}\right)$, the second term in the right hand side of (39) converge to 0 as $n \rightarrow \infty$ and it is not hard to prove by using the dominated convergence theorem the term in the left hand side of (39) converges. Thus, we conclude that $\lim _{n \rightarrow \infty} \int_{\mathbb{R}^{d}} \int_{s}^{T} f\left(r, x, u_{n}(r, x)\right) \phi_{t}(r, x) d r d x$ exists. Moreover by the monotonocity condition of $f$ and the same arguments as in step 2 of the proof of theorem 4.1, we get for all compact support function $\phi \in C_{c}^{2}\left(\mathbb{R}^{d}\right)$

$$
\begin{aligned}
& \int_{\mathbb{R}^{d}} \int_{s}^{T} u(r, x) d \phi_{t}(r, x) d x+\left(u(s, \cdot), \phi_{t}(s, \cdot)\right)-\left(g(\cdot), \phi_{t}(\cdot, T)\right)+\int_{s}^{T} \mathcal{E}\left(u(r, \cdot), \phi_{t}(r, \cdot)\right) d r \\
= & \int_{\mathbb{R}^{d}} \int_{s}^{T} f(r, x, u(r, x)) \phi_{t}(r, x) d r d x .
\end{aligned}
$$

Now we consider the case when $f$ depends on $\nabla u$ and satisfies the assumption 2.3 with (iii) replaced by (12). Like in the step 3 of the proof of theorem 4.1, we construct a mapping $\Psi$ from $\mathcal{H}$ into itself. Then by this mapping, we define a sequence $\left\{u_{n}\right\}$ in $\mathcal{H}$, beginning with a matrix-valued function $v^{0} \in \mathbf{L}^{2}\left([0, T] \times \mathbb{R}^{n \times d}, d t \otimes \rho(x) d x\right)$. Since $f\left(t, x, u, v^{0}(t, x)\right)$ satisfies the assumptions of step 2, the $\operatorname{PDE}\left(g, f\left(t, x, u, v^{0}(t, x)\right)\right)$ admits a unique solution $u_{1} \in \mathcal{H}$. For $n \in \mathbb{N}$, denote

$$
u_{n}(t, x)=\Psi\left(u_{n-1}(t, x)\right),
$$

i.e. $u_{n}$ is the weak solution of the $\operatorname{PDE}\left(g, f\left(t, x, u, \sigma^{*} \nabla u_{n-1}(t, x)\right)\right)$. Set $\widetilde{u}_{n}(t, x):=u_{n}(t, x)-$ $u_{n-1}(t, x)$. In order to estimate the difference, we introduce the corresponding $\operatorname{BSDE}\left(g, f_{n}\right)$ for $n=1$, where $f_{n}(t, x, u)=f\left(t, x, u, \nabla u_{n-1}(t, x)\right)$. So we have $Y_{s}^{n, t, x}=u_{n}\left(s, X_{s}^{t, x}\right), Z_{s}^{n, t, x}=$ $\sigma \nabla u_{n}\left(s, X_{s}^{t, x}\right)$. Then we apply the Itô's formula to $\left|\widetilde{Y}^{n, t, x}\right|^{2}$, where $\tilde{Y}_{s}^{n, t, x}:=Y_{s}^{n, t, x}-Y_{s}^{n-1, t, x}$. With the equivalence of the norms, similarly as in step 3 , for $\gamma=1+\frac{2 k_{1}^{2}}{k_{2}^{2}} k^{2}$, we have

$$
\begin{aligned}
& \int_{\mathbb{R}^{d}} \int_{t}^{T} e^{\gamma s}\left(\left|\widetilde{u}_{n}(s, x)\right|^{2}+\left|\sigma^{*} \nabla\left(\widetilde{u}_{n}\right)(s, x)\right|^{2}\right) \rho(x) d s d x \\
\leqslant & \left(\frac{1}{2}\right)^{n-1} \int_{\mathbb{R}^{d}} \int_{t}^{T} e^{\gamma s}\left(\left|\widetilde{u}_{2}(s, x)\right|^{2}+\left|\sigma^{*} \nabla\left(\widetilde{u}_{2}\right)(s, x)\right|^{2}\right) \rho(x) d s d x \\
\leqslant & \left(\frac{1}{2}\right)^{n-1}\left(\left\|u_{1}(s, x)\right\|_{\gamma}^{2}+\left\|u_{2}(s, x)\right\|_{\gamma}^{2}\right) .
\end{aligned}
$$

where $\|u\|_{\gamma}^{2}:=\int_{\mathbb{R}^{d}} \int_{t}^{T} e^{\gamma s}\left(|u(s, x)|^{2}+\left|\sigma^{*} \nabla u(s, x)\right|^{2}\right) \rho(x) d s d x$, which is equivalent to the norm $\|\cdot\|$ of $\mathcal{H}$. So $\left\{u_{n}\right\}$ is a Cauchy sequence in $\mathcal{H}$, it admits a limit $u$ in $\mathcal{H}$, and by the fixed point theorem, $u$ is a solution of the $\operatorname{PDE}(g, f)$. 
Then for each $n \in \mathbb{N}$, we have for $\phi \in C_{c}^{2}\left(\mathbb{R}^{d}\right)$

$$
\begin{aligned}
& \int_{\mathbb{R}^{d}} \int_{s}^{T} u_{n}(r, x) d \phi_{t}(r, x) d x+\left(u_{n}(s, \cdot), \phi_{t}(s, \cdot)\right)-\left(g(\cdot), \phi_{t}(\cdot, T)\right)+\int_{s}^{T} \mathcal{E}\left(u_{n}(r, \cdot), \phi_{r}(r, \cdot)\right) d r \\
= & \int_{\mathbb{R}^{d}} \int_{s}^{T} f\left(r, x, u_{n}(r, x), \sigma^{*} \nabla u_{n-1}(r, x)\right) \phi_{t}(r, x) d r d x \\
= & \int_{\mathbb{R}^{d}} \int_{s}^{T} f\left(r, x, u_{n}(r, x), \sigma^{*} \nabla u(r, x)\right) \phi_{t}(r, x) d r d x \\
& +\int_{\mathbb{R}^{d}} \int_{s}^{T}\left[f\left(r, x, u_{n}(r, x), \sigma^{*} \nabla u_{n-1}(r, x)\right)-f\left(r, x, u_{n}(r, x), \sigma^{*} \nabla u(r, x)\right)\right] \phi_{t}(r, x) d r d x .
\end{aligned}
$$

Noticing that $f$ is Lipschitz in $z$, we get

$$
\left|f\left(r, x, u_{n}(r, x), \sigma^{*} \nabla u_{n-1}(r, x)\right)-f\left(r, x, u_{n}(r, x), \sigma^{*} \nabla u(r, x)\right)\right| \leqslant k\left|\sigma^{*} \nabla u_{n-1}(r, x)-\sigma^{*} \nabla u(r, x)\right| .
$$

So the last term of the right side converges to 0 , since $\left\{\sigma^{*} \nabla u_{n}\right\}$ converges to $\sigma^{*} \nabla u$ in $\mathbf{L}^{2}([0, T] \times$ $\left.\mathbb{R}^{d}, d t \otimes \rho(x) d x\right)$. Now we are in the same situation as in the first part of proof, and in the same way, we deduce that the following holds: for $\phi \in C_{c}^{2}\left(\mathbb{R}^{d}\right)$

$$
\begin{aligned}
& \int_{\mathbb{R}^{d}} \int_{s}^{T} u(r, x) d \phi_{t}(r, x) d x+\left(u(s, \cdot), \phi_{t}(s, \cdot)\right)-\left(g(\cdot), \phi_{t}(\cdot, T)\right)+\int_{s}^{T} \mathcal{E}\left(u(r, \cdot), \phi_{t}(r, \cdot)\right) d r \\
= & \int_{\mathbb{R}^{d}} \int_{s}^{T} f\left(r, x, u(r, x), \sigma^{*} \nabla u(r, x)\right) \phi_{t}(r, x) d r d x, d t \otimes d x, \text { a.s.. }
\end{aligned}
$$

Now if $f$ satisfies assumption 2.3, we know that $u$ is solution of the $\operatorname{PDE}(g, f)$ if and only if $\widehat{u}=e^{\mu t} u$ is solution of the $\operatorname{PDE}(\widehat{g}, \widehat{f})$, where

$$
\widehat{g}(x)=e^{\mu T} g(x), \widehat{f}(t, x, y, x)=e^{\mu t} f\left(t, x, e^{-\mu t} y, e^{-\mu t} z\right)-\mu y,
$$

and $\widehat{f}$ satisfies assumption 2.3-(iii) replaced by (12). So we know now: for $\phi \in C_{c}^{2}\left(\mathbb{R}^{d}\right)$,

$$
\begin{aligned}
& \int_{\mathbb{R}^{d}} \int_{s}^{T} \widehat{u}(r, x) d \phi_{t}(r, x) d x+\left(\widehat{u}(s, \cdot), \phi_{t}(s, \cdot)\right)-\left(\widehat{g}(\cdot), \phi_{t}(\cdot, T)\right)+\int_{s}^{T} \mathcal{E}\left(\widehat{u}(r, \cdot), \phi_{t}(r, \cdot)\right) d r \\
= & \int_{\mathbb{R}^{d}} \int_{s}^{T} \widehat{f}(r, x, \widehat{u}(r, x), \nabla \widehat{u}(r, x)) \phi_{t}(r, x) d r d x, d t \otimes d x, \text { a.s.. }
\end{aligned}
$$

Notice that $d\left(e^{\mu r} u(r, x)\right)=\mu e^{\mu r} u(r, x) d r+e^{\mu r} d(u(r, x))$, so by the integration by parts formula (for stochastic process), we get

$$
\begin{aligned}
& \int_{\mathbb{R}^{d}} \int_{s}^{T} u(r, x) d \phi_{t}(r, x) d x \\
= & \int_{\mathbb{R}^{d}} \int_{s}^{T} e^{-\mu r} \widehat{u}(r, x) d \phi_{t}(r, x) d x d r \\
= & e^{-\mu T}\left(\widehat{g}(\cdot), \phi_{t}(\cdot, T)\right)-e^{-\mu s}\left(\widehat{u}(s, \cdot), \phi_{t}(s, \cdot)\right)+\mu \int_{s}^{T} e^{-\mu r} \int_{\mathbb{R}^{d}} \widehat{u}(r, x) \phi_{t}(r, x) d x d r \\
& -\int_{s}^{T} \int_{\mathbb{R}^{d}} e^{-\mu r} \phi_{t}(r, x)[\mathcal{L} \widehat{u}(r, x)+\widehat{f}(r, x, \widehat{u}(r, x), \nabla \widehat{u}(r, x))] d r d x .
\end{aligned}
$$


Using (11), we get that for $\phi \in C_{c}^{2}\left(\mathbb{R}^{d}\right)$,

$$
\begin{aligned}
\int_{\mathbb{R}^{d}} \int_{s}^{T} u(r, x) d \phi_{t}(r, x) d x= & \left(g(\cdot), \phi_{t}(\cdot, T)\right)-\left(u(s, \cdot), \phi_{t}(s, \cdot)\right)-\int_{s}^{T} \int_{\mathbb{R}^{d}} \phi_{t}(r, x) \mathcal{L} u(r, x) d r d x \\
& +\int_{s}^{T} \int_{\mathbb{R}^{d}} \phi_{t}(r, x) f(r, x, u(r, x), \nabla u(r, x)) d r d x \\
= & \left(g(\cdot), \phi_{t}(\cdot, T)\right)-\left(u(s, \cdot), \phi_{t}(s, \cdot)\right)-\int_{s}^{T} \mathcal{E}\left(u(r, \cdot), \phi_{t}(r, \cdot)\right) d r \\
& +\int_{s}^{T} \int_{\mathbb{R}^{d}} \phi_{t}(r, x) f(r, x, u(r, x), \nabla u(r, x)) d r d x,
\end{aligned}
$$

and finally, the result follows.

\subsection{Some a priori estimates}

In this subsection, we consider the non-markovian Reflected BSDE associated to $(\xi, f, L)$ :

$$
\left\{\begin{array}{l}
Y_{t}=\xi+\int_{t}^{T} f\left(t, Y_{s}, Z_{s}\right) d s+K_{T}-K_{t}-\int_{t}^{T} Z_{s} d B_{s} \\
Y_{t} \geqslant L_{t} \\
\int_{0}^{T}\left(Y_{s}-L_{s}\right) d K_{s}=0
\end{array}\right.
$$

under the following assumptions :

(H1) a final condition $\xi \in \mathbf{L}^{2}\left(\mathcal{F}_{T}\right)$,

(H2) a coefficient $f: \Omega \times[0, T] \times \mathbb{R} \times \mathbb{R}^{d} \rightarrow \mathbb{R}$, which is such that for some continuous increasing function $\varphi: \mathbb{R}_{+} \longrightarrow \mathbb{R}_{+}$, a real numbers $\mu$ and $C>0$ :

(i) $\quad f(\cdot, y, z)$ is progressively measurable, $\forall(y, z) \in \mathbb{R} \times \mathbb{R}^{d}$;

(ii) $|f(t, y, 0)| \leqslant|f(t, 0,0)|+\varphi(|y|), \forall(t, y) \in[0, T] \times \mathbb{R}$, a.s.;

(iii) $E \int_{0}^{T}|f(t, 0,0)|^{2} d t<\infty$;

(iv) $\left|f(t, y, z)-f\left(t, y, z^{\prime}\right)\right| \leqslant C\left|z-z^{\prime}\right|, \forall(t, y) \in[0, T] \times \mathbb{R}, z, z^{\prime} \in \mathbb{R}^{d}$, a.s.

(v) $\left(y-y^{\prime}\right)\left(f(t, y, z)-f\left(t, y^{\prime}, z\right)\right) \leqslant \mu\left(y-y^{\prime}\right)^{2}, \forall(t, z) \in[0, T] \times \mathbb{R}^{d}, y, y^{\prime} \in \mathbb{R}$, a.s.

(vi) $y \rightarrow f(t, y, z)$ is continuous, $\forall(t, z) \in[0, T] \times \mathbb{R}^{d}$, a.s.

(H3) a barrier $\left(L_{t}\right)_{0 \leqslant t \leqslant T}$, which is a continuous progressively measurable real-valued process, satisfying

$$
E\left[\varphi^{2}\left(\sup _{0 \leqslant t \leqslant T}\left(e^{\mu t} L_{t}^{+}\right)\right)\right]<\infty
$$

and $\left(L_{t}^{+}\right)_{0 \leqslant t \leqslant T} \in \mathbf{S}^{2}(0, T), L_{T} \leqslant \xi$, a.s.

We shall give an a priori estimate of the solution $(Y, Z, K)$ with respect to the terminal condition $\xi$, the coefficient $f$ and the barrier $L$. Unlike the Lipshitz case, we have in addition the term $E \varphi^{2}\left(\sup _{0} \leqslant t \leqslant T\left(L_{t}^{+}\right)\right)$and a constant, which only depends on $\varphi, \mu, k$ and $T$ : 
Proposition 6.1. There exists a constant $C$, which only depends on $T, \mu$ and $k$, such that

$$
\begin{aligned}
E\left[\sup _{0 \leqslant t \leqslant T}\left|Y_{t}\right|^{2}+\int_{0}^{T}\left|Z_{s}\right|^{2} d s+\left|K_{T}\right|^{2}\right] \leqslant & C E\left[\xi^{2}+\int_{0}^{T} f^{2}(t, 0,0) d t+\varphi^{2}\left(\sup _{0 \leqslant t \leqslant T}\left(L_{t}^{+}\right)\right)\right] \\
& +C E\left[\sup _{0 \leqslant t \leqslant T}\left(L_{t}^{+}\right)^{2}+1+\varphi^{2}(2 T)\right] .
\end{aligned}
$$

Proof. Applying Itô's formula to $\left|Y_{t}\right|^{2}$, and taking expectation, then

$$
\begin{aligned}
E\left[\left|Y_{t}\right|^{2}+\int_{t}^{T}\left|Z_{s}\right|^{2} d s\right] & =E\left[|\xi|^{2}+2 \int_{t}^{T} Y_{s} f\left(s, Y_{s}, Z_{s}\right) d s+2 \int_{t}^{T} L_{s} d K_{s}\right. \\
& \leqslant E\left[|\xi|^{2}+2 \int_{t}^{T} Y_{s} f(s, 0,0) d s+2 \int_{t}^{T}\left(\mu\left|Y_{s}\right|^{2}+k\left|Y_{s}\right|\left|Z_{s}\right|\right) d s+2 \int_{t}^{T} L_{s} d K_{s}\right] .
\end{aligned}
$$

It follows that

$$
E\left[\left|Y_{t}\right|^{2}+\frac{1}{2} \int_{t}^{T}\left|Z_{s}\right|^{2} d s\right] \leqslant E\left[|\xi|^{2}+2 \int_{t}^{T} f^{2}(s, 0,0) d s+\left(2 \mu+1+2 k^{2}\right) \int_{t}^{T}\left|Y_{s}\right|^{2} d s+2 \int_{t}^{T} L_{s} d K_{s}\right] .
$$

Then by Gronwall's inequality, we have

$$
E\left|Y_{t}\right|^{2} \leqslant C E\left[|\xi|^{2}+\int_{0}^{T} f^{2}(s, 0,0) d s+\int_{0}^{T} L_{s} d K_{s}\right]
$$

then

$$
E \int_{0}^{T}\left|Z_{s}\right|^{2} d s \leqslant C E\left[|\xi|^{2}+\int_{0}^{T} f^{2}(s, 0,0) d s+\int_{0}^{T} L_{s} d K_{s}\right]
$$

where $C$ is a constant only depends on $\mu, k$ and $T$, in the following this constant can be changed line by line.

Now we estimate $K$ by approximation. By the existence of the solution, theorem 2.2 in Lepeltier et al. [12], we take the process $Z$ as a known process. Without losing generality we write $f(t, y)$ for $f\left(t, y, Z_{t}\right)$, here $f(t, 0)=f\left(t, 0, Z_{t}\right)$ is a process in $\mathbf{H}^{2}(0, T)$. Set

$$
\begin{aligned}
\xi^{m, n} & =(\xi \vee(-n)) \wedge m, \\
f^{m, n}(t, y) & =f(t, y)-f(t, 0)+(f(t, 0) \vee(-n)) \wedge m .
\end{aligned}
$$

For $m, n \in \mathbf{N}, \xi^{m, n}$ and $\sup _{0 \leqslant t \leqslant T} f^{m, n}(t, 0)$ are uniformly bounded. Consider the $\operatorname{RBSDE}\left(\xi^{m, n}, f^{m, n}, L\right)$,

$$
\begin{aligned}
& Y_{t}^{m, n}=\xi^{m, n}+\int_{t}^{T} f^{m, n}\left(t, Y_{s}^{m, n}\right) d s+K_{T}^{m, n}-K_{t}^{m, n}-\int_{t}^{T} Z_{s}^{m, n} d B_{s}, \\
& Y_{t}^{m, n} \geqslant L_{t}, \int_{0}^{T}\left(Y_{s}^{m, n}-L_{s}\right) d K_{s}^{m, n}=0 .
\end{aligned}
$$

if we recall the transform in step 2 of the proof of theorem 2.2 in Lepeltier et al. [12], since $\xi^{m, n}$, $f^{m, n}(t, 0) \leqslant m$, we know that $\left(Y_{t}^{m, n}, Z_{t}^{m, n}, K_{t}^{m, n}\right)$ is the solution of this RBSDE, if and only if $\left(Y^{m, n \prime}, Z^{m, n \prime}, K^{m, n \prime}\right)$ is the solution of $\operatorname{RBSDE}\left(\xi^{m, n \prime}, f^{m, n \prime}, L^{\prime}\right)$, where

$$
\left(Y_{t}^{m, n \prime}, Z_{t}^{m, n \prime}, K_{t}^{m, n \prime}\right)=\left(Y_{t}^{m, n}+m(t-2(T \vee 1)), Z_{t}^{m, n}, K_{t}^{m, n}\right)
$$


and

$$
\begin{aligned}
\xi^{m, n \prime} & =\xi^{m, n}+2 m T-m(T \vee 1), \\
f^{m, n \prime}(t, y) & =f^{m, n}(t, y-m(t-2(T \vee 1)))-m, \\
L_{t}^{\prime} & =L_{t}+m(t-2(T \vee 1)) .
\end{aligned}
$$

Without losing generality we set $T \geqslant 1$. Then $\xi^{m, n \prime} \leqslant 0$ and $f^{m, n \prime}(t, 0) \leqslant 0$. Since $\left(Y^{m, n \prime}, Z^{m, n \prime}, K^{m, n \prime}\right)$ is the solution of $\operatorname{RBSDE}\left(\xi^{m, n \prime}, f^{m, n \prime}, L^{\prime}\right)$, then we have

$$
K_{T}^{m, n \prime}=Y_{0}^{m, n \prime}-\xi^{m, n \prime}-\int_{0}^{T} f^{m, n \prime}\left(s, Y_{s}^{m, n \prime}, Z_{s}\right) d s+\int_{0}^{T} Z_{s}^{m, n \prime} d B_{s}
$$

which follows

$$
E\left[\left(K_{T}^{m, n \prime}\right)^{2}\right] \leqslant 4 E\left[\left|Y_{0}^{m, n \prime}\right|^{2}+\left|\xi^{m, n \prime}\right|^{2}+\left(\int_{0}^{T} f^{m, n \prime}\left(s, Y_{s}^{m, n \prime}\right) d s\right)^{2}+\int_{0}^{T}\left|Z_{s}^{m, n \prime}\right|^{2} d s\right] .
$$

Applying Itô's formula to $\left|Y^{m, n}\right|^{2}$, like (40) and (41), we have

$$
E\left|Y_{t}^{m, n}\right|^{2}+E \int_{t}^{T}\left|Z_{s}^{m, n}\right|^{2} d s \leqslant C E\left[\left|\xi^{m, n}\right|^{2}+\int_{t}^{T}\left(f^{m, n}(s, 0)\right)^{2} d s+\int_{t}^{T} L_{s} d K_{s}^{m, n}\right] .
$$

So

$$
\begin{aligned}
\left|Y_{0}^{m, n \prime}\right|^{2}+\int_{0}^{T}\left|Z_{s}^{m, n \prime}\right|^{2} d s & =2\left|Y_{0}^{m, n}\right|^{2}+8 m^{2} T^{2}+E \int_{0}^{T}\left|Z_{s}^{m, n}\right|^{2} d s \\
& \leqslant C E\left[\left|\xi^{m, n}\right|^{2}+\int_{0}^{T}\left(f^{m, n}(s, 0) d s\right)^{2}+\int_{0}^{T} L_{s} d K_{s}^{m, n}\right]+8 m^{2} T^{2} .
\end{aligned}
$$

For the third term on the right side of (42), from Lemma 2.3 in Lepeltier et al. [12], we remember that

$$
\left(\int_{0}^{T} f^{m, n \prime}\left(s, Y_{s}^{m, n \prime}\right) d s\right)^{2} \leqslant \max \left\{\left(\int_{0}^{T} f^{m, n \prime}\left(s, \widetilde{Y}_{s}^{m, n}\right) d s\right)^{2},\left(\int_{0}^{T} f^{m, n \prime}\left(s, \bar{Y}_{s}^{m, n}\right) d s\right)^{2}\right\},
$$

where $\left(\widetilde{Y}^{m, n}, \widetilde{Z}^{m, n}\right)$ is the solution the following BSDE

$$
\widetilde{Y}_{t}^{m, n}=\xi^{m, n \prime}+\int_{0}^{T} f^{m, n \prime}\left(s, \widetilde{Y}_{s}^{m, n}\right) d s-\int_{0}^{T} \widetilde{Z}_{s}^{m, n} d B_{s}
$$

and

$$
\bar{Y}_{s}^{m, n}=e s s \sup _{\tau \in \mathcal{T}_{t, T}} E\left[\left(L_{\tau}^{\prime}\right)^{+} 1_{\{\tau<T\}}+\left(\xi^{m, n}\right)^{+} 1_{\{\tau=T\}} \mid \mathcal{F}_{t}\right]=e s s \sup _{\tau \in \mathcal{T}_{t, T}} E\left[\left(L_{\tau}^{\prime}\right)^{+} \mid \mathcal{F}_{t}\right] .
$$

From (44), and proposition 2.2 in Pardoux [13], we have

$$
\begin{aligned}
E\left(\int_{0}^{T} f^{m, n \prime}\left(s, \tilde{Y}_{s}^{m, n}\right) d s\right)^{2} & \leqslant C E\left[\left|\xi^{m, n \prime}\right|^{2}+\left(\int_{0}^{T} f^{m, n \prime}(s, 0) d s\right)^{2}\right] \\
& \leqslant C E\left[\left|\xi^{m, n}\right|^{2}+\int_{0}^{T}\left(f^{m, n}(s, 0)\right)^{2} d s\right]+C \varphi^{2}(2 m T)+C m^{2}
\end{aligned}
$$


For the other term in (43), with $\sup _{0 \leqslant t \leqslant T} \bar{Y}_{s}^{m, n}=\sup _{0 \leqslant t \leqslant T}\left(L_{t}^{\prime}\right)^{+}$, we get

$$
\begin{aligned}
E\left(\int_{0}^{T} f^{m, n \prime}\left(s, \bar{Y}_{s}^{m, n}\right) d s\right)^{2} & \leqslant E\left[\int_{0}^{T} 2\left(f^{m, n \prime}(s, 0)\right)^{2} d s+2 T \varphi^{2}\left(\sup _{0 \leqslant t \leqslant T}\left(L_{t}^{\prime}\right)^{+}\right]\right. \\
& \leqslant E\left[4 \int_{0}^{T} f^{m, n}(s, 0)^{2} d s+2 T \varphi^{2}\left(\sup _{0 \leqslant t \leqslant T}\left(L_{t}\right)^{+}\right)\right]+2 m^{2} T+4 T \varphi^{2}(2 m T) .
\end{aligned}
$$

Consequently, we deduce that

$$
\begin{aligned}
E\left[\left(K_{T}^{m, n}\right)^{2}\right]= & E\left[\left(K_{T}^{m, n \prime}\right)^{2}\right] \\
\leqslant & C E\left[\left|\xi^{m, n}\right|^{2}+\int_{0}^{T}\left(f^{m, n}(s, 0)\right)^{2} d s+\int_{t}^{T} L_{s} d K_{s}^{m, n}+\varphi^{2}\left(\sup _{0 \leqslant t \leqslant T}\left(L_{t}\right)^{+}\right)+m^{2}+\varphi^{2}(2 m T)\right] \\
\leqslant & C E\left[|\xi|^{2}+\int_{0}^{T}\left(f\left(s, 0, Z_{s}\right)\right)^{2} d s+\varphi^{2}\left(\sup _{0 \leqslant t \leqslant T}\left(L_{t}\right)^{+}\right)+\sup _{0 \leqslant t \leqslant T}\left(\left(L_{t}\right)^{+}\right)^{2}\right]+\frac{1}{2} E\left[\left(K_{T}^{m, n}\right)^{2}\right] \\
& +C\left(m^{2}+\varphi^{2}(2 m T)\right) .
\end{aligned}
$$

Moreover using (41) and the fact that $f$ is Lipschitz on $z$, it follows that

$$
\begin{aligned}
E\left[\left(K_{T}^{m, n}\right)^{2}\right] \leqslant & C E\left[|\xi|^{2}+\int_{0}^{T}(f(s, 0,0))^{2} d s+\varphi^{2}\left(\sup _{0 \leqslant t \leqslant T}\left(L_{t}\right)^{+}\right)+\sup _{0 \leqslant t \leqslant T}\left(\left(L_{t}\right)^{+}\right)^{2}\right. \\
& \left.+\int_{0}^{T} L_{s} d K_{s}\right]+C\left(m^{2}+\varphi^{2}(2 m T)\right) .
\end{aligned}
$$

Let $m \rightarrow \infty$, then

$$
E\left[\left|\xi^{m, n}-\xi^{n}\right|^{2}\right] \rightarrow 0, E \int_{0}^{T}\left|f^{m, n}(t, 0)-f^{n}(t, 0)\right|^{2} \rightarrow 0,
$$

where $\xi^{n}=\xi \vee(-n)$ and $f^{n}(t, y)=f(t, y)-f(t, 0)+f(t, 0) \vee(-n)$

Thanks to the convergence result of step 3 of the proof for theorem 2.2 in [12], we know that $\left(Y^{m, n}, Z^{m, n}, K^{m, n}\right) \rightarrow\left(Y^{n}, Z^{n}, K^{n}\right)$ in $\mathbf{S}^{2}(0, T) \times \mathbf{H}_{d}^{2}(0, T) \times \mathbf{A}^{2}(0, T)$, where $\left(Y^{n}, Z^{n}, K^{n}\right)$ is the soultion of the $\operatorname{RBSDE}\left(\xi^{n}, f^{n}, L\right)$. Moreover $K_{T}^{m, n} \searrow K_{T}^{n}$ in $\mathbf{L}^{2}\left(\mathcal{F}_{T}\right)$, so we have $K_{T}^{n} \leqslant K_{T}^{1, n}$, which implies for each $n \in \mathbf{N}$,

$$
E\left[\left(K_{T}^{n}\right)^{2}\right] \leqslant E\left[\left(K_{T}^{1, n}\right)^{2}\right]
$$

Then, letting $n \rightarrow \infty$, by the convergence result in step 4 , since

$$
E\left[\left|\xi^{n}-\xi\right|^{2}\right] \rightarrow 0, E \int_{0}^{T}\left|f^{n}(t, 0)-f(t, 0)\right|^{2} \rightarrow 0,
$$

the sequence $\left(Y^{n}, Z^{n}, K^{n}\right) \rightarrow(Y, Z, K)$ in $\mathbf{S}^{2}(0, T) \times \mathbf{H}_{d}^{2}(0, T) \times \mathbf{A}^{2}(0, T)$, where $(Y, Z, K)$ is the 
solution of the $\operatorname{RBSDE}(\xi, f, L)$. From (46), and (45) for $m=1$, we get

$$
\begin{aligned}
E\left[\left(K_{T}\right)^{2}\right] \leqslant & C E\left[|\xi|^{2}+\int_{0}^{T}(f(s, 0,0))^{2} d s+\varphi^{2}\left(\sup _{0 \leqslant t \leqslant T}\left(L_{t}\right)^{+}\right)+\sup _{0 \leqslant t \leqslant T}\left(\left(L_{t}\right)^{+}\right)^{2}\right. \\
& \left.+\int_{0}^{T} L_{s} d K_{s}\right]+C\left(1+\varphi^{2}(2 T)\right) \\
\leqslant & C E\left[|\xi|^{2}+\int_{0}^{T}(f(s, 0,0))^{2} d s+\varphi^{2}\left(\sup _{0 \leqslant t \leqslant T}\left(L_{t}\right)^{+}\right)+\sup _{0 \leqslant t \leqslant T}\left(\left(L_{t}\right)^{+}\right)^{2}\right] \\
& +\frac{1}{2} E\left[\left(K_{T}\right)^{2}\right]+C\left(1+\varphi^{2}(2 T)\right) .
\end{aligned}
$$

Then it follows that for each $t \in[0, T]$,

$$
\begin{aligned}
E\left[\left|Y_{t}\right|^{2}+\int_{0}^{T}\left|Z_{s}\right|^{2} d s+\left(K_{T}\right)^{2}\right] \leqslant & C E\left[|\xi|^{2}+\int_{0}^{T}(f(s, 0,0))^{2} d s+\varphi^{2}\left(\sup _{0 \leqslant t \leqslant T}\left(L_{t}\right)^{+}\right)\right. \\
& \left.+\sup _{0 \leqslant t \leqslant T}\left(\left(L_{t}\right)^{+}\right)^{2}\right]+C\left(1+\varphi^{2}(2 T)\right) .
\end{aligned}
$$

Finally we get the result, by applying BDG inequality.

Acknowledgements. We would like to thank the anonyme referee of this paper for his interesting comments.

\section{References}

[1] Bally V., Caballero E., El-Karoui N. and B. Fernandez : Reflected BSDE's PDE's and Variational Inequalities (to appear in Bernoulli 2007).

[2] Barles, G. and L. Lesigne : SDE, BSDE and PDE. In: El Karoui, N. and Mazliak, L., (Eds.), Backward Stochastic differential Equatons. Pitman Research Notes in Mathematics Series, 364, 47-80 (1997). MR1752675

[3] Bally, V. and A. Matoussi : Weak solutions for SPDEs and Backward doubly stochastic differential equations, Journal of Theoretical Probability, Vol. 14, No. 1, 125-164 (2001). MR1822898

[4] Bensoussan A. and Lions J.L. : Applications des Inéquations variationnelles en controle stochastique. Dunod, Paris (1978).

[5] J.M. Bismut : Conjugate Convex Functions in Optimal Stochastic Control, J. Math. Anal. Appl., 44, 384-404 (1973). MR0329726

[6] El Karoui, N., Peng S., Quenez M.C. : Backward Stochastic Differential Equations in Finance, Mathematical Finance, 7, 1-71 (1997). MR1434407

[7] El Karoui, N., Kapoudjian, C., Pardoux, E., Peng S. and M.C. Quenez: Reflected Solutions of Backward SDE and Related Obstacle Problems for PDEs, Ann. Probab. 25, no 2, 702-737 (1997). MR1434123 
[8] El Karoui, N., Pardoux, E., Quenez, M.C. : Reflected backward SDE's and American options. Numerical Method in Finance, Cambridge University Press, 215-231 (1997) MR1470516

[9] Hamadène S. and Ouknine, Y. : Reflected backward stochastic differential equation with jumps and random obstacle. Electronic Journal of Probability, Vol. 8, 1-20 (2003). MR1961164

[10] Hamadène S., Lepeltier, J.-P. and Matoussi A. : Double barriers reflected backward SDE's with continuous coefficients. Pitman Research Notes in Mathematics Series, 364 , 115-128 (1997).

[11] H. Kunita : Stochastic differential equations and stochastic flows of diffeomorphisms. Ecole dété de Probabilité de Saint-Flour, Lect. Notes Math., 1097, 143-303 (1982). MR0876080

[12] Lepeltier, J.P., Matoussi, A. and M. Xu : Reflected BSDEs under monotonicity and general increasing growth conditions. Advanced Applied Probability, 37, 134-159 (2005). MR2135157

[13] E. Pardoux : BSDE's, weak convergence and homogenization of semilinear PDE's in Nonlinear analysis, Differential Equations and Control, F. H. Clarke \& R. J. Stern Eds, Kluwer Acad. Pub., 503-549, (1999). MR1695013

[14] Pardoux, E. and Peng, S. : Adapted Solutions of Backward stochastic differential equation, Systems and Control Letters, 14, 55-61 (1990). MR1037747

[15] Pardoux, E. and S. Peng. : Backward SDEs and Quasilinear PDEs. In Rozovskii, B. L. and Sowers, R.B. (eds.), Stochastic Partial Differential Equations and Their Applications, LNCIS 186, Springer (1992). MR1176785

[16] Peng, S. : Probabilistic interpretation for system of quasilinear parabolic partial differential equations. Stochastics and stochastics reports, Vol. 37, pp 61-74.(1991) MR1149116

[17] Revuz D. and Yor, M. : Continuous martingales and Brownian motion (Springer, Berlin) (1991). MR1083357

[18] Yong, J. and Zhou, X. Y. : Stochastic Controls. Hamiltonian Systems and HJB Equations. Springer Verlag, New York (1999). MR1696772 